\title{
Die Chondriosomen als Träger erblicher Anlagen. Cytologische Studien am Hühnerembryo.
}

\author{
Von \\ Friedrich Meves in Kiel. \\ Hierzu Tafel XXXIX-XLII. \\ Inhaltsverzeichnis.
}

I. Die nucleare Vererbungstheorie als Gegenstand der Kritik.

II. Stellungnahme verschiedener Autoren (Boveri, O. Hertwig, C. Rabl, Strasburger) gegenüber den aufgezählten Ergebnissen der Entwicklungsmechanik.

III. Liber die Lokalisation einer Erbsubstanz im Cytoplasma.

IV. Die Chondriosomen in den Zellen des Hühnerembryos von der zweiten Hälfte des ersten bis zum Beginn des vierten Tages der Bebrütung.

a) Untersuchungsmethode.

b) Darstellung der Befunde.

V. Die Chondriosomen in ihrem Verhältnis zur Filarmasse Flemmings. Über Protoplasmastruktur.

VI. Die Rolle der Chondriosomen bei der Zelldifferenziernng und Vererbung.

VII. Die Chondriosomen als das materielle Substrat der Naegelischen Idioplasmatheorie.

VIII. Über die Frage, wie weit die Chondriosomen den von der Kernsul)stanz in ihrer Eigenschaft als Erbmasse erfüllten Bedingungen genügen.

IX. Über zwei neuere Versuche, das Vererbungsmonopol des Kerns zu erweisen.

\section{Die nucleare Vererbungstheorie als Gegenstand der Kritik.}

Im Jahre 1875 kam O. Hertwig im ersten Teil seiner „Beitrăge zur Kenntnis der Bildung, Befruchtung und Teilung der tierischen Eier" zu dem Resultat, dass die Befruchtung auf der Kopulation oder Verschmelzung eines weiblichen Eikerns mit einem männlichen Samenkern beruht.

Diese Ansicht stiess anfangs bei den meisten Forschern auf Widerspruch. Strasburger (1877, S. 509) erweiterte sie dahin, dass nicht bloss die Zellkerne, sondern überhaupt die gleichwertigen Teile der kopulierenden Zellen sich im Geschlechtsakt vereinigten und dass hierin das Wesen der Befruchtung bestehe. 
Hensen (1881, S. 126) bezeichnete die Auffassung O. Hertwigs insofern als eine glückliche, als sie unsere Kenntnisse von dem Befruchtungsvorgang vertiefe, , indem sie zu den bisher nur in Betracht gezogenen chemischen und physikalischen Momenten noch hinzufügt das für die Lebenserscheinungen (und die Vererbung) so bedeutsame morphologische Moment, dass nämlich die Materie in bestimmter Formung mitwirkt ; er betonte aber (S. 127), dass ,jedenfalls neben der Kernmasse des Zoosperms auch protoplasmatische Substanz in das Ei eingeht, was zu vernachlăssigen kein Grund vorliegt".

Nussbaum (1883) studierte die Befruchtung von Ascaris megalocephala und betrachtete sie (S. 217) als eine „Konjugation zweier Zellen", „deren Protoplasma mit allen aus ihm hervorgegangenen Bildungen sich vermischt, deren Kerne nach Ausstossung der Richtungskörper sich vereinigen und den Kern des befruchteten Eies darstellen": (s. auch denselben 1884, S. 182).

Auch Van Beneden (1883, S. 397) erklarte auf Grund seiner am gleichen Objekt angesteliten Lintersuchungen, dass nichts zu der Versicherung berechtige, dass die Rolle des Spermaprotoplasmas bei der Befruchtung eine sekundäre sei; immerhin gäbe es einige Tatsachen, welche seine Bedeutung zweifelhaft erscheinen liessen.

Flemming (1882, 1 S. 34) hatte auf Grund von Befunden, welche eine Bestätigung derjenigen von O. Hertwig und Fol waren, den Satz aufgestellt, dass sich im Furchungskern das Chromatin sowohl eines mannlichen als eines weiblichen Kerngebildes vereinigt. In einem Referat „Über Bauverhältnisse, Befruchtung und erste Teilung der tierischen Eizelle" im Biologischen Centralblatt (Jan. 1884, Bd. 3, S. 682) weist er darauf hin, dass dieser Satz mit der 'These 0 . Hertwigs: „Die Befruchtung beruht auf der Verschmelzung ron geschlechtlich differenzierten Zellkernen “ allerdings nicht ganz identisch sei. Wit den Worten "berult auf* sei wohl etwas mehr gesagt, als sich beute vertreten lasse. „Denn das Spermatozoon bringt ausser dem Kerı (Kopf) auch Zellsubstanz (Schwanz und Nittelstück) mit ins $\mathrm{Ei}$ und lässt auch sie möglicherweise in ihm aufgehen, und es lässt sich nicht ausschliessen, dass die Befruchtung zugleich hierauf mitberuhen kann. "*

Im Jahre 1884 erschien dann $\mathrm{N}$ a eg elis glänzendes, auf scharfsimniger spekulation gegründetes Werk, „Mechanisch-physiologische Theorie der Abstammungslehre", in welchem er in den Keimzellen 
auf Grund theoretischer Erwägungen ein "Anlageplasma“ oder „Idioplasma“, welches der Träger der erblichen Anlagen ist, von dem ïbrigen Plasma unterschied. Das Idioplasma gehört zu dem festen Plasma oder Stereoplasma; denn "die Vererbung bei del geschlechtlichen Fortpflanzung lässt nur die eine Erklarung $z u$, dass die Anlagen bloss durch feste (unlösliche), nicht durch gelöste Stoffe übertragen werden". Die spezifischen Eigenschaften des Idioplasmas werden durch seine Zusammensetzung aus kleinsten Teilen (Micellen) bedingt. Die Überlegungen, welche Na egeli dazu geführt haben, Idioplasma und gewöhnliches Plasma zu unterscheiden, sind folgende: "Ich habe beide“, sagt er (S.27), „als verschieden angegeben, weil mir dies der einfachste und natürlichste Weg scheint, um die ungleichen Beziehungen der Plasmasubstanzen zu den erblichen Anlagen zu begreifen, wie sie bei der geschlechtlichen Fortpflanzung deutlich werden. An die befruchtete und entwicklungsfăhige Eizelle hat die Mutter hundert- oder tausendmal mehr Plasmasubstanzen, in denselben aber keinen grösseren Anteil an erblichen Eigenschaften geliefert als der Vater. Wenn das unbefruchtete $\mathrm{Ei}$ ganz aus Idioplasma bestünde, so würde man nicht begreifen, warum es nicht entsprechend seiner Masse in dem Kinde wirksam wăre, warum dieses nicht immer in ganz überwiegendem Grade der Mutter ahnlich würde. Besteht die spezifische Eigentümlichkeit des Idioplasmas in der Anordnung und Beschaffenheit der Micelle, so lăsst sich eine gleich grosse Erbschaftsübertragung nur denken, wenn in den bei der Befruchtung sich vereinigenden Substanzen gleichviel Idioplasma enthalten ist, und der überwiegende Erbschaftsanteil, der bald von der Mutter, bald vom Vater herstammen soll, muss dadurch erklärt werden, dass bald in der unbefruchteten Eizelle, bald in den mit derselben sich vereinigenden Spermatozoiden eine grössere Menge von Idioplasma sich befindet. Bestehen die Spermatozoide bloss aus Idioplasma, so enthalten die nicht befruchteten Eizellen bis auf 999 Promille nicht idioplasmatisches Stereoplasma."

An Na egelis Werk anknüpfend, haben darauf O. Hertwig und Strasburger unabhängig voneinander noch im Jahre 1884 auf Grund neuer Untersuchungen über die Befruchtung die Theorie aufgestellt, dass das "Idioplasma" im Chromatin der Kerne zu suchen sei; der Zellsubstanz könne keine Bedeutung für die Vererbung zugeschrieben werden. 
Diese Lehre wurde nunmehr von Weismann (1885), Koelliker (1885), Hensen (1885), Van Beneden (1887), Weigert (1887), Boveri (1889) und zahlreichen anderen angenommen.

Jedoch erhoben auch fernerhin verschiedene Autoren Widerspruch, mit der immer wiederholten Mahnung, auch das Protoplasma nicht zu vernachlässigen.

Zuerst war es Nussbaum (1886, S. 517), welcher schrieb: ,Mit Rücksicht auf die in neuerer Zeit vielseitig veränderte Auffassung von dem Wesen der Befruchtung möchte ich an dieser Stelle ganz besonders darauf hinweisen, dass Ei- und Samenelement stets ganze Zellen sind, und sowohl Kern als Protoplasma der beiden Zellen sich kopulieren. Wo man wie bei Ascaris megalocephala das Eindringen des Samenkörpers ins Ei und die folgenden Verïnderungen mit befriedigender Sicherheit verfolgen kann, findet man die Beweisstücke für diesen Satz, und selbst die Beobachtungen Strasburgers an Phanerogamen dürten die Frage wohl nicht im entgegengesetzten Sinne entscheiden. Ehe wir die neueren Methoden zum Nachweise der Kerne besassen, glaubten viele, es genüge das Protoplasma zur Befruchtung allein. Jetzt hat sich zugunsten des Kernes ein Umschwung vollzogen. Vielleicht können wir aber dereinst auch an minder günstigen Objekten, im Besitz geeigneter Methoden, die Bedeutung des Protoplasmas bei der Befruchtung des Eies erkennen."

Frenzel (1886) gab es als wahrscheinlich $z u$, dass bei der geschlechtlichen Fortpflanzung von männlichen Keimstoffen nur das Chromatin zur Wirkung kommt; sicher bewiesen sei dies aber bis heute nicht, da sowohl andere Kernbestandteile (Kernsaft) wie auch protoplasmatische Bestandteile dabei in Frage kommen könnten.

Ra u ber (1887, S. 170) sprach die Überzeugung aus, „dass dem Kern nicht ausschliesslich das Vererbungsgeschaft zufällt, sondern nur teilweise. Dasselbe gilt von dem Protoplasma. Auch dieses hat teilweise Vererbungsfunktionen. Die totale Vererbung aber wird bewirkt durch beide Teile zusammen, durch den Kern und durch das Protoplasma." "Ich halte somit", sagt R a u ber, „den Standpunkt für einen einseitigen, der das Ganze der Vererbung allein dem Kern aufbürden will." 
W a ld e y e r (1888, S. 94) erkennt an, dass für die Lehre, nach welcher die Kerne als alleinige Träger der Vererbungssubstrate anzusehen sind, schwerwiegende Gründe in Menge vorgebracht werden können, gibt aber zu bedenken, dass ein entscheidender Beweis noch nicht geliefert sei. "Dieser wäre erst dann geliefert, wenn wir für eine Spermatozoenart absolut sicher wüssten, dass in ihre Konstitution kein Teil des Protoplasmas der betreffenden Bildungszelle übergeht, oder dass, falls auch Protoplasma überginge, dieses nicht in den Befruchtungsvorgang einbezogen würde." Den gleichen Standpunkt vertrat er 1893 in einer den damaligen Stand des Problems zusammenfassenden Rede, mit welcher er die Verhandlungen der Anatomischen Gesellschaft in Göttingen eröffinete.

Nach Verworn (1891) ist alles, was für die nucleare Vererbungstheorie ins Feld geführt werden kann, einer sorgfaltigen Kritik gegenüber hinfallig. Mit welchem Recht man stets das Protoplasma des Spermatozoons, das immer mit in die Befruchtung eingeht, unberücksichtigt bei Seite lässt, ist ihm unbegreiflich. In der ganzen Natur findet man das Gesetz realisiert, dass die Fortpflanzung und damit die Vererbung nur auf Übertragung von Kern und Protoplasma der Zelle auf ihre Abkömmlinge beruht. Andererseits besteht die Tatsache, dass von einer Zelle weder das Protoplasma ohne Kern, noch (wie Verworn gezeigt hat) der Kern ohne Protoplasma dauernd lebensfähig ist. Kern und Protoplasma sind beide an dem Stoffwechsel der Zelle beteiligt und für sein Bestehen unentbehrlich. Wenn man den Stoffwechsel als den eigentlichen elementaren Lebensvorgang betrachtet, dessen Ausdruck die Lebenserscheinungen sind, so sind auch alle vererbten Eigentümlichkeiten nur Ausdruck des Stoffwechsels. Was sich vererbt, das ist die für jeden Organismus eigentümliche Art des Stoffwechsels. Protoplasma und Kern sind beide Träger der Vererbungssubstanzen und die Vererbung kommt nur zustande durch Übertragung von Substanz beider Teile und ibrer Stoffwechselbeziehungen auf die Nachkommen.

Inzwischen waren die $\mathrm{Z}$ e $\mathrm{nt} \mathrm{r}$ alkörper oder Centrosomen in den Vordergrund des Interesses getreten.

0. Hertwig (1884), vor allem aber Boveri (1887) hatten bereits geschlossen, dass der Samenfaden ausser einem mannlichen 
Kern auch ein männliches Polkörperchen oder einen Zentralkörper ins Ei hineinträgt. An eine vererbende Wirkung der Zentralkörper wurde aber erst gedacht, nachdem Fol (1891) am Seeigelei den Nachweis erbracht zu liaben glaubte, dass ein Cytozentrum vom Spermatozoon abstammt, ein anderes der Eizelle angehört, dass beide sich teilen und je eine männliche und weibliche Teilungshälfte zu je einem Polkörperchen der ersten Teilung zusammentreten.

Ausser Fol selbst hat besonders R. S. Bergh (Febr. 1892, S. 50; dat. Nov. 1891) auf die Möglichkeit hingewiesen, dass die Zentralkörper bei der Vererbung beteiligt sind, und sich gleichzeitig gegen diejenigen Forscher gewandt, welche den Kern als den alleinigen Trăger der Vererbung ansprechen.

Auch Fick (Nov. 1892 sowie 1893), welcher den Import eines Cytozentrums beim Axolotl beobachtete, betonte, dass die „Kernsubstanz des Spermatozoons nicht allein als die männliche Vererbungssubstanz angesehen werden könne."

Die "Centrenquadrille" Fols fand nun aber bei einer Nachuntersuchung durch Wilson und Mathews (18!95), Boveri (1895) und andere keine Bestatigung. Was wir ferner, zuerst durch meine Untersuchungen (1897-1899), iiber die Art und Weise kennen lernten, wie die Cytozentren beim Aufbau der Spermien umgewandelt und zerlegt werden, war gleichfalls der Anschauung nicht günstig, dass sie bei der Übertragung erblicher Eigenschaften beteiligt sind.

So kehrte man dazu zurück, allgemein auf das Protoplasma hinzuweisen. Der Hinweis auf dieses aber verstärkte sich immer mehr infolge der zahlreichen Ergebnisse der experimentellen Forschung, welche energisch an der nuclearen Vererbungstheorie rüttelten.

Eine Reihe von Autoren führten an befruchteten, aber noch ungefurchten Eiern Anschnit tversuche aus, bei denen der Zellkern intakt blieb; trotzdem traten Defektbildungen bei den sich entwickelnden Larven auf.

Driesch und Morgan (1896) zeigten, dass Entnahme von Plasma am Ctenophorenei seitliche Defekte an den Larven bedingen kann. 
Crampton (1896) exstirpierte am Ei eines marinen Prosobranchiers, Ilyanassa, vor oder wahrend des Einschneidens der ersten Furchung den sogenannten Dotterlappen und fand, dass dadurch die Mesoblastbildung verhindert wird.

Fischel (1903) entfernte am ungefurchten oder im ersten Furchungsstadium begriffenen Ctenophorenei einen bestimmten Teil des Dotters und konstatierte, dass bei den sich entwickelnden Larven eine oder mehrere Rippen ausfielen; bei Ausschaltung anderer bestimmter Teile des Zellleibs blieben andere bestimmte Teile des Larvenkörpers unentwickelt.

Ähnliche Experimente stellte Wils on (1904) an den Eiern besonders von Mollusken (Patella und Dentalium) an.

Alle diese Versuche, bei welchen der Kern, wie gesagt, unverletzt blieb, wiesen auf das Protoplasma als den Sitz der embryonalen Differenzierung hin und zwar führten sie zu dem Schluss, dass in den Eiern mehrere verschiedene Plasmaarten vorhanden sind, welche bestimmte Beziehungen zur Bildung bestimmter Organe besitzen.

Dieser Schluss schien damit übereinzustimmen, dass sich in den Eiern zahlreicher Tiere tatsächlich auf Grund ron Farbung oder Lichtbrechung mehrere Substanzen unterscheiden lassen, welche bei der Furchung in bestimmte Zellen ïbergehen.

So fand z. B. Driesch (1897, S. 116), dass im Plasma des Myzostoma-Eies drei durch ihre Farbung unterschiedene Substanzen enthalten sind; von diesen wird die eine, rotgefarbte bei der Furchung in die Micromeren, eine zweite glasartige in die Entomeren, die dritte grünlich schwarze resp milchige in die Somatoblasten übergeführt.

Conklin (1905) konnte am Ei von Cynthia nicht weniger als sechs verschiedene Plasmaarten erkennen, welche in ihrer definitiven Lage schon zu einer merkwürdig frühen Periode der Eireifung lokalisiert sind. Sie können hier mit Leichtigkeit und Sicherheit durch Reifung und Befruchtung, Furchung, Gastrulation und spätere Stadien verfolgt werden, bis sie bestimmten Organen oder Teilen der Larve Ursprung geben.

Schliesslich scheinen auch die Erfahrungen über Bastardierung durchaus zu beweisen, dass die Vererbung der Hauptsache nach auf protoplasmatischer Basis beruht. 
Boveri war schon 1892 (S. 469) auf Grund von Kreuzungsversuchen zwischen zwei verschiedenen Seeigelarten zu dem damals nur kurz mitgeteilten Ergebnis gelangt, dass „der Furchungsprozess durch die Anordnung des Eimaterials allein vollkommen bestimmt zu sein scheint."

Weitere Bastardierungsversuche, die von Driesch (1898, 1903) und Boveri (1903) angestellt wurden, ergaben als übereinstimmendes Resultat, dass die Furchungsgeschwindigkeit und der allgemeine Habitus der Blastulae rein mütterliche Charaktere besitzen, also ausschliesslich von der Beschaffenheit des Eies abhängig sind.

Loeb (1903) gelang es, Seeigeleier mit dem Samen von Seesternen $z u$ befruchten; die sich entwickelnden Larven zeigten rein mütterliche Charaktere. Nach Loeb (1906, S. 277) „hat es sich mehr und mehr herausgestellt, dass, was an Präformation des Embryo im Ei vorhanden ist, nicht im Kern, sondern im Protoplasma des Eies zu suchen ist."

Godlewski (1906) befruchtete Seeigeleier mit dem Sperma eines Crinoiden; auch er erhielt Larven von rein mütterlichem Habitus. Ferner glückte es ihm, kernlose Eifragmente eines Seeigels mit Crinoidensperma zu befruchten und auch dabei Embryonen (Gastrulae) zu erhalten, welche rein mütterliche Charaktere zur Schau trugen. „Daraus geht, will man die Ergebnisse der Versuche noch so vorsichtig deaten, zum wenigsten hervor, dass bis zum Gastrulastadium, ohne das Vorhandensein des mütterlichen Kerns, mütterliche Charaktere zum Vorschein kommen können (Godlewski, 1906, S. 639).

\section{Stellungnahme verschiedener Autoren (Boveri, O. Hertwig, C. Rabl, Strasburger) gegenüber den auf- gezählten Ergebnissen der Entwicklungsmechanik.}

Zwingen nun in der Tat speziell die zuletzt erwähnten Tatsachen der experimentellen Forschung, die Lehre, dass die Kerne die alleinigen Träger der erblichen Eigenschaften sind, fallen $z u$ lassen? Von verschiedenen Seiten wird diese Frage auch heute noch mehr oder weniger bestimmt verneint.

Boveri (1907, S. 246 u. folg.; auch schon 1892, S. 469, 1903, S. 354 und 1904, S. 111) hat sich über die Rolle von Kern 
und Protoplasma bei der Vererbung folgende Anschauung gebildet. Er bezeichnet es als selbstverständlich, dass die im Ei gegebenen Faktoren, welche zusammenwirken müssen, damit ein neues Individuum von gleicher Art entsteht wie das elterliche, jedenfalls zum einen Teil im Prot oplas m a liegen. Allein die Frage, um welche es sich bei dem Vererbungsproblem handelt, ist diese: wie ist es zu erklären, dass trotz des ungeheuren Übergewichts, welches das Ei im protoplasmatischen Anteil der Vererbungsfaktoren besitzt, das neue Individuum doch dem Tater ganz ebenso ahnlich sehen kann wie der Mutter? Hierfür kommt nach Boveri das Protoplasma des Eies nicht in Betracht; die Übertragung der spezifischen Merkmale von den Eltern auf das Kind geschieht ausschliesslich durch die Chromosomen van Ei- und Spermakern.

Auf diesem Wege gelangt Boveri zu der Vorstellung, dass in der Entwicklung zwei in bezug auf die Mitwirkung des Kerns essentiell verschiedene Perioden zu unterscheiden sind: eine erste, in der die Konstitution des Eiplasmas massgebend ist, während von den Chromosomen nur gewisse generelle Qualitäten wirksam sind; und eine zweite, in welcher die Chromosomen durch ibre spezifischen Eigenschaften zur Geltung kommen.

Dass die erste Entwicklungsperiode durch die Konstitution des Eiplasmas bestimmt wird, erscheint deshalb höchst wahrscheinlich. weil sich bei Bastardierungen alle Merkmale dieser ersten Periode als rein mütterlich darstellen; dadurch, dass sich im Plasma des unbefruchteten Eies gewisse Primitivorgane vorbereitet finden, darf es sogar bis zu einem gewissen Grad als sicher bewiesen gelten.

In der $z$ weite $n$ Periode, in welcher die Chromosomen durch ihre spezifischen Eigenschaften wirken, geht der Kern zugrunde, wenn die Wirkung der Chromosomen ausbleibt oder eine unrichtige ist. Godlewski hat aus den kernlosen Fragmenten von Echinuseiern, die er mit Antedonsamen befruchtete, trotz zahlreicher Versuche nur Larven jüngerer Stadien erhalten; die vier bestentwickelten Keime starben auf dem Gastrulastadium ab. Nach B overi ist mit diesem Stadium eben die ausserste Grenze erreicht, bis zu der Eiplasma eines Echiniden mit Chromosomen eines Crinoiden sich entwickeln kann.

Auch O. Hertwig (1906, S. 562) halt in seiner "Allgemeinen Biologie" an der Hypothese fest, dass in der Kernsubstanz das 
Idioplasma oder der als Träger der erblichen Eigenschaften wirksamste Teil der Zelle zu suchen sei. Damit wird, wie er sagt (S. 563), „gar nicht geleugnet, dass bei der Entstehung eines Organismus das im Ei enthaltene Protoplasma oder soweit solches noch im Samenfaden zugegen sein sollte, auch dieses seine Eigenschaften direkt vererbt". Dies erscheint nach O. Hertwig sogar selbstverständlich, wenn man die von de Vries aufgestellte Hypothese der "intracellulären Pangenesis" akzeptiert, nach welcher von den sogenannten "Pangenen", die in den Kernen meist in einem inaktiven Zustand als Erbmasse vereinigt sind, einige aktiv werden und dabei in das Protoplasma der Zelle einwandern sollen.

Hierzu möchte ich, unter Hinweis auf Waldeyer (1893, S. 9), bemerken, dass durch diese Hypothese das uns interessierende Problem nicht aus der Welt geschafft wird. Nach de Vries hätte „das Protoplasma seine Erbmasse erst in zweiter Linie erhalten, ursprünglich wäre sie doch in den Kernen enthalten gewesen:" Die Frage ist jedoch die, „ob wir dem Protoplasma ebenso originär wie den Kernen Erbmasse zuschreiben dürfen, oder nicht".

Nach C. R a bl (1906) müssen wir, wenn wir über die Grundprinzipien der Entwicklung und damit zugleich über das Wesen der Vererbung Klarheit bekommen wollen, stets folgendes im Auge behalten. Kern und Protoplasma stehen in materieller oder substantieller Wechselwirkung zueinander. Aufgabe und Abgabe von Stoffen sind in der Zelle genau lokalisiert, an bestimmte, anatomisch wohl charakterisierte Regionen des Protoplasmas und des Kerns gebunden.

Das unreife Ei, die Eibildungszelle, bildet während der langen Dauer der Wachstumsperiode solche Plasmasubstanzen, die, wenn sie auch noch nicht als organbildende bezeichnet werden dürfen, doch zu deren späterer Bildung unbedingt erforderlich sind. Diese verschiedenen Plasmaarten sind innerhalb des Eies in bestimmter Weise lokalisiert.

Auf die langdauernde Periode des Wachstums folgt die kurze der Reifung. Während oder nach Ablauf des Reifungsprozesses dringt das Spermatozoon in das Ei ein. Ei- und Spermakern treten nicht sofort in Teilung, sondern nehmen den Umweg durch ein sogenanntes Ruhestadium (ein Kernreticulum). Vit diesem Stadium beginnt eine lebhafte Wechselwirkung zwischen den Substanzen der beiden Vorkerne oder des aus der Verschmelzung 
beider hervorgegangenen Keimkerns oder ersten Furchungskerns mit den während der Wachstumsperiode des Eies im Bildungsdotter entstandenen Plasmaarten, und das Produkt dieser Wechselwirkung sind eben die organbildenden Substanzen, deren Lokalisation sich unmittelbar von der Lokalisation der verschiedenen Plasmaqualitäten des unreifen Eies ableitet.

„Nun erklären sich die mannigfachen Ergebnisse der ... Experimente in durchaus befriedigender und ungezwungener Weise. Entfernt man vor oder während des Einschneidens der ersten Furche, also zu einer Zeit, zu der im Ei bereits die ersten organbildenden Substanzen gebildet sind, bestimmte Teile des Dotters, so muss es zu ganz bestimmten Ausfallserscheinungen kommen; stört man sie in ihrer Lage, so müssen später die Organe, zu deren Entwicklung sie in Beziehung stehen, eine entsprechende Lageveründerung zeigen." In den Echinuseiern, die von Godlewski zu seinen Versuchen verwendet worden sind, hat mütterliche Kiernsubstanz bereits auf das Eiplasma eingewirkt. Hierzu kommt, dass die Befruchtung (mit Antedonsperma) in Seewasser ausgeführt wurde, dem etwas Natronlauge zugesetzt war: Godlewski selbst gibt an, dass dadurch die Lebensfähigkeit sowohl der Eier als der Spermatozoen beeinträchtigt werde. "Wie sehr die spermatozoen unter der Einwirkung von Natronlauge leiden, kann man leicht sehen, wenn man z. B. Froschsperma damit behandelt. Uabei schwellen die Köpfe mächtig an und ihr Chromatin quillt und wird in mehr oder weniger tiefgreifender Weise verändert. Man kann sich dem Eindruck nicht verschliessen, dass solches Chromatin in seiner Kraft geschwächt, dass es nicht mehr imstande ist, den normalen Einfluss auf das Eiplasma auszuüben und lernt so verstehen, dass die organbildenden Substanzen, die unter seinem Eintluss entstehen, den väterlichen Charakter nicht mehr rein oder überhaupt nicht mehr zum Ausdruck bringen."

„Jedenfalls beweisen diese Versuche, dass die organbildenden Substanzen oder "Anlagesubstanzen" oder, wie man sie sonst nennen will, keineswegs, wie dies jetzt fast allgemein angenommen wird, ausschliesslich vom Kern geliefert werden.“ - „Wie die Dinge heute liegen, können wir selbst die Beteiligung der in der Regel sehr geringen Menge Protoplasmas, welche das Spermatozoon in das Ei mitbringt, an der Vererbung nicht ohne weiteres ausschliessen." 
Strasburger hat zu den von Godlewski angestellten Bastardierungsversuchen 1907, 1 und 2 und 1908 Stellung genommen. Nach ihm machen sich dabei bestimmende Einflüsse des Cytoplasmas in besonders auffalliger Weise geltend. „Übt doch selbst die Beschaffenheit der äussern Umgebung eine nachweisbare Wirkung auf den Entwicklungsgang aus. Wenn somit in einem hybriden Keim nur die Eigenschaften des einen Elters zur Geltung kommen, so kann das sehr wohl dadurch veranlasst sein, dass das von der Mutter stammende Cytoplasma die văterlichen Chromosomen an ihrer Äusserung hindert" (1907, 1, S. 124). Dagegen beweist es nach Strasburger nicht, „dass die Wirkungssphäre der Spermatozoiden einzuschränken sei: und dass die Chromosomen nicht die einzigen Träger der erblichen Eigenschaften seien“ (1907, 2, S. 508). Stra sburger fügt hinzu, „dass bestimmte Sonderungen zu Beginn der Keimentwicklung auch durch mechanische Ursachen mitbedingt sein könnten, also eine spezifische Äusserung der im Kern vertretenen Erbfähigkeiten nicht verlangen“ (1908, S. 536; vergl. auch 1907, 1, S. 124).

\section{III. Über die Lokalisation einer Erbsubstanz im Cytoplasma.}

Die Anschauung, dass nur der Kern bei der Vererbung wirksam sei, ist zunächst hauptsächlich dadurch entstanden, dass bei Befruchtung und Teilung des Eies der Kern allein verfolgbar blieb, wahrend das durch den Samenfaden eingeführte Protoplasma als differentes Element zu verschwinden schien. Eine andere Substanz, die man als Idioplasma im Sinne Naegelis ansprechen konnte, schien demnach überhaupt nicht $\mathrm{zu}$ existieren. Auch bis heute ist es noch nicht gelungen, eine Beteiligung cytoplasmatischer Bestandteile des Spermiums (abgesehen vom Cytozentrum) bei der Befruchtung direkt nachzuweisen. Bei dem berechtigten Einfluss, den die Sichtbarkeit der Dinge auf Deutung und Spekulation ausübt, kann es daher nicht wundernehmen, dass zahlreiche Autoren auch heute noch an dem „Vererbungsmonopol" des Kerns festhalten.

Hierzu kommt, dass das Chromatin sich in Ei- und Samenzelle äquivalent gegenübersteht, dass es auf die aus dem befruchteten Ei hervorgehenden Zellen in gleicher Weise verteilt wird und dass es vor der Befruchtung eine "Reduktion" erfährt. 
Insofern entspricht es durchaus Forderungen, welche man an die Vererbungssubstanz zu stellen berechtigt scheint.

Es ist klar. dass, wenn neben dem (hromatin eine cyto)plasmatische Vererbungssubstanz überhaupt existiert. wir sie am leichtesten in den Spermien bezw. ihren Bildungszellen werden auffinden müssen. Denn diese enthalten auf kleinstem Raum alle zur ľbertragung der vaterlichen Eigenschaften notwendigen Stoffe, wïlurend in dem meistens sehr voluminösen Ei ihre Auffindung durch die zablreichen, darin enthaltenen Reservestoffe (Fettkügelchen, Dotterplăttchen) erschwert sein wird.

Natürlich braucht nicht jeder cytoplasmatische Bestandteil der Spermien Vererbungspotenzen zu besitzen. Die Fibrillen des Axenfadens haben möglicherweise ausschliesslich die Bedeutung ron Bewegungsorganen: die Gebilde am vorderen Kopfende, welche aus dem Idiozom oder der Centrotheca hervorgehen, dienen vielleicht ebenso ausschliesslich als ein Bohr-oder Schneideapparat beim Eindringen der Spermien in die Eier. Dass den C'entriolen der Spermien eine vererbende Kraft zukommt, ist aus Gründen, die ich oben angeführt babe, unwabrscheinlich. -

Ratselhaft in funktioneller Beziehung war bisher ein Bestandteil der Spermatiden, welcher am liangsten, seit 1867, bei Insekten als "Nebenkörper* (v. la Valette St. George) oder als "Nebenkern“ (Bütschli, 1871) bekannt ist. Der Yebenkern kommt bei der Umbildung der Spermatide in das Spermium hinter dem Kopf zu liegen und wird zur Ümbüllung des intrazellulären Teils des Schwanzes, bezw. des aus den Centriolen hervorgehenden Mittelstückes verwendet.

Seine Entstehung ist zuerst von v. la Valet te St. Ge orge (1886) bei Blatta aufgeklart. v. la Valette St. George konstatierte, dass der Nebenkern der Spermatiden sich aus Körnern, „Cytomikrosomen“, bildet, welche auch schon in den Spermatocyten vorhanden sind.

Die gleichen Körner sind später von verschiedenen Untersuchern, 7. B. von Henking (91) und Toyama (94), gesehen worden.

In den Spermatiden der Maus hatte A. v. Brunn schon vorher (1884) ,Protoplasmagranula:" beschrieben, von denen er 
feststellt, dass sie bei der Bildung des Spermiums in den Spiralfaden des Verbindungsstückes übergehen.

Benda fand dieselben Körner 1897 ebenfalls bei der Maus und bei Phalangista, 1898 bei einer grösseren Reihe anderer Wirbeltiere (Säuger, Sauropsiden, Amphibien) und schilderte ihr Verhalten bei der Histogenese der Spermien. Er führte den Namen ,Mitochondrien" für die Körner ein und erklärte, dass sie „ein neues, vielleicht einer spezifischen Funktion dienendes Zellorgan" darstellen.

Weiter zeigte Benda $(1899,1)$ bei vielen Wirbeltieren und verschiedenen Wirbellosen, dass die Mitochondrien sich in sämtlichen Generationen der samenbildenden Zellen finden. In derselben Arbeit untersuchte er auch andere Zellarten auf das Vorkommen von Mitochondrien und gewann dabei den Eindruck, „dass alle protoplasmareichen Zellen die entsprechend färbbaren und entsprechend angeordneten Körner wenigstens spurenweise enthalten ". Er konstatierte ibr Vorhandensein in jugendlichen quergestreiften Muskelfasern, in glatten Muskelzellen (zwischen den Fibrillen), in Flimmerzellen, in Leukocyten und Knochenmarkszellen, in den Sertolischen und interstitiellen Zellen des Hodens, in Nierenepithelien; weiter $(1899,2)$ in den Ovarialeiern und Zellen älterer Blastulastadien von Triton sowie in den Eiund Follikelzellen der Maus.

Ich selbst (1900) habe den Nachweis geführt, dass die Mitochondrien Bendas mit den von v. la Valet te St. George beschriebenen "Cytomikrosomen" identisch sind. Daraus ergab sich, „dass der Spiralfaden der Säugetiere der von dem Nebenkern gebildeten Schwanzumhüllung bei Wirbellosen homolog ist" (Meves, 1900, S. 586). Sodann habe ich das Verhalten der Mitochondrien wăhrend der Mitose in den Spermatocyten von Paludina und Pygaera genau studiert.

Was nun die physiologische Bedeutung der Mitochondrien anlangt, so hatte Benda $(1899,1)$ die Hypothese ausgesprochen, dass sie ein motorisches Organ der Zelle darstellen. Ich habe aber schon 1899 einen Versuch ausgeführt, welcher zeigt, dass diese Annahme unzutreffend ist. Ich fand nämlich, dass man an lebenden Salamandersamenfaden das von Mitochondrien freie Endstück mehr oder weniger weit hinter seinem Anfang abschneiden kann, ohne dass der Flossensaum dieses Abschnitts seine 
Bewegung einbüsst (vergl. Me ves 1900, S. 600). B e n d a (1903, S. 780) bezeichnet diese Beobachtung zwar als "etwas problematisch". 1) Ich halte aber an ihrer Richtigkeit durchaus fest und betrachte damit diese Hypothese Bendas als widerlegt.

Dagegen kann ich einer ,anderen physiologischen Erwägung“", mit welcher Bend a sein Referat in den „Ergebnissen der Anatomie und Entwicklungsgeschichte " (1903) schliesst, nur beipflichten. Benda sagt: Die von den Mitochondrien gebildete Hülle ,liegt stets in dem Abschnitt des Spermiums, welcher unzweifelhaft bei der Befruchtung mit in das Ei dringt. Bei dem Ascarisspermium ist höchst wahrscheinlich der gestreifte Abschnitt, der voran in das Ei wandert, chondriogener Abkunft. Nach den Beobachtungen R. Ficks am Axolotl, L. Michaelis' am Triton, Van der Strichts bei der Fledermaus, Henkings bei Insekten, v. Kostaneckis bei Physa treten die Geisselabschnitte, die den chondriogenen Mantel besitzen, mit in die Sphärenstrahlung. In den Blastomeren von Triton . . . habe ich reichliche Mengen von Mitochondrien gefunden, aber es ist mir noch nicht gelungen, den entscheidenden Augenblick der Spermienumbildung abzufassen. Trotzdem ist mit Bestimmtheit vorauszusagen, dass die Mitochondrien, ebenso wie sie individualisiert die Mitosen überdauern, auch als individualisierte Bestandteile der männlichen Geschlechtszelle innerhalb der weiblichen wiedererscheinen und an der Befruchtung teilnehmen werden. Diese Feststellung, die mir als das dringendste Postulat erscheint, würde den Schlussstein in der Kennzeichnung der Mitochondrien als Zellorgan

1) B en da (l.c.) sagt: ,Soweit ich an meinen Färbungen sehe, reicht die chondriogene Hülle fast so weit, wie der Flossensaum. Es müsste also erst durch Färbung eines solchen Stückes mit sicherer Beweglichkeit der Nachweis erbracht werden, dass es wirklich keinen chondriogenen Mantel mehr enthielt." Demgegenüber verweise ich auf die Fig. 6 von $\mathrm{Cz}$ ermak (Gesammelte Schriften, Bd. 1. Taf. 3, Leipzig 1879) oder auf meine Fig. 48 (Arch. f. mikr. Anat., Bd. 50, Taf. VIII), welche auf den ersten Blick erkennen lassen, dass der Protoplasmamantel (welcher die Mitochondrien enthält) n i cht fast so weit als der Flossensaum reicht. Der Protoplasmamantel hört eine weite Strecke vor dem hintersten Ende des Achsenfadens auf; den von Protoplasma entblössten Teil des Achsenfadens bezeichnet man ja eben als „Endstiick". Der Flossensaum dagegen reicht bis an das hinterste Ende des Endstückes; sein „Randfaden" ragt sogar noch um eine Strecke weit darüber hinaus. 
abgeben und einem dem Zellleib angehörenden Bestandteil die Rolle eines der Faktoren der Vererbung vindizieren, da das Vorhandensein der gleichen Gebilde in den weiblichen Geschlechtszellen von $\mathrm{mir}$ bereits unzweifelhaft beobachtet ist."

Ich selbst bin 1907, 2 durch cytologische Beobachtungen an Wirbeltierembryonen dazu gekommen, die Meinung auszusprechen, dass die Mitochondrien als Vererbungsträger fungieren.

Ich fand, dass Gebilde, welche sich mit den zur Darstellung der Mitochondrien geeigneten Methoden intensiv färben, bei jungen Embryonen von Huhn und Saugetieren ausnabmslos in sämtlichen Zellen vorhanden sind. Sie erscheinen nur selten als Körner, Mitochondrien, viel häufiger als Stäbe oder als mehr oder weniger lange, meistens gewundene glatte Fäden, welche in ibrem ganzen Verlauf gleich dick sind.

Stabförmige oder fadenförmige Mitochondrienkörper waren uns bis dabin hauptsächlich von sich teilenden Samenzellen bekannt, bei denen sie im Umkreis der Spindel gelagert sind. Ich hatte sie in meinen früheren Arbeiten als Chondriomiten bezeichnet, wurde aber dann darauf aufmerksam, dass unter diesem Namen nach Benda Reihen von Mitochondrien verstanden werden sollen, die in "Plasmafäden" eingefügt sind. Ich schlug daher 1907, 2 vor, Stäbe oder Fäden, die ausschliesslich aus Mitochondriensubstanz bestehen, "Chondriokonten" zu nennen (von xorrcs, lat. contus, Stange, Stab). Beide Arten von Bildungen, Mitochondrien und Chondriokonten, möchte ich unter der Bezeichnung "Chondriosomen" zusammenfassen.

Ich vermochte nun zu konstatieren, dass die Mitochondrien bezw. Chondriokonten, welche in den embryonalen Zellen vorhanden sind, die Anlagesubstanz für die verschiedensten Faserstrukturen, z. B. Myofibrillen, Neurofibrillen, Neurogliafasern, Bindegewebsfasern bilden. Sodann konnte es auch nach meiner Meinung kaum zweifelhaft sein, dass die Mitochondrien an der Befruchtung teilnehmen, d. h. dass die Chondriosomen der embryonalen Zellen teils von der männlichen, teils von der weiblichen Geschlechtszelle abstammen. Auf diese Weise wurde ich zu dem Schlusse gedrängt, dass die Chondriosomen eine cy toplasmatische Vererbungssubstanz repräsentieren. -

Die Befunde, die ich an dieser Stelle mitteilen will, betreffen ausschliesslich das Vorkommen der Chondriosomen beim 
Hühnerembryo von der zweiten Hălfte des ersten bis zum Beginn des vierten Tages der Bebrütung.

IV. Die Chondriosomen in den Zellen des Hühnerembryos von der zweiten Halfte des ersten bis zum Beginn des vierten Tages der Bebrütung.

a) Untersuchungsmethode.

Zur Fixierung der Embryonen habe ich hauptsăchlich eine modifizierte Flemming sche Chromosmiumessigsăure angewandt. Das sog. starke Flemmingsche Gemisch bewirkt bei zarten Objekten, so auch bei jungen Embryonen, nicht selten eine nicht unerhebliche Schrumpfung, welche offenbar auf seinem Gehalt an $1 \%$ iger Chromsaure beruht. Ich habe daher bei früheren Arbeiten vielfach ein starkes Gemisch gebraucht, welches mit dem gleichen Quantum destillierten Wassers verdünnt war. Da auf diese Weise aber auch der Gehalt an Osmiumsäure eine Halbierung erfährt, habe ich es neuerdings vorgezogen, mir ein Gemisch mit 1/2\% iger Chromsäure herzustellen. $\mathrm{Zu}$ der Chromsäure babe ich einen Zusatz von $1 \%$ Kochsalz gemacht, wovon ich bei meinen Arbeiten über Amphibienblut gute Resultate gesehen $z u$ haben glaube. Ausserdem habe ich, der wichtigen Empfehlung Bendas folgend, den Gehalt an Essigsäure für die Chondriosomendarstellung auf ein Minimum beschränkt. Die von mir benutzte Lösung hat demnach folgende Zusammensetzung:

$1 / 2 \%$ ige Chromsäure (mit Zusatz von $1 \%$ Kochsalz) $15 \mathrm{ccm}$ $2 \%$ ige Osmiumsăure . . . . . . . . . 3-4" Eisessig . . . . . . . . . . . . . . 3-4 Tropfen.

Für ganz junge Keimscheiben (bis zur ca. 36. Stunde) hat mir F lemming sche Lösung in Form des schwachen Gemisches ${ }^{1}$ ) (mit Zusatz von $1 \%$ Kochsalz) ebenfalls gute Resultate ergeben.

Zur Färbung der Chondriosomen habe ich mich dieses $\mathrm{Mal}$ in erster Linie der Eisenhämatoxylinmethode nach $\mathbf{M}$. Heidenhain bedient; bezüglich der Art und Weise, wie ich hierbei vorgehe, verweise ich auf eine frühere Arbeit (1907, 1, S. 417-418). Hier möchte ich nur bemerken, dass es erforderlich ist, eine altere, gut ausgereifte Hämatoxylinlösung zu verwenden.

1) $1 \%$ ige Chromsäure $25 \mathrm{ccm}, 1 \%$;oige Osmiumsäure $10 \mathrm{ccm}, 1 \%$ ige Essigsäure $10 \mathrm{ccm}$, dest. Wasser $55 \mathrm{ccm}$. 
Mit Hilfe dieses Verfahrens (Fixierung mit Flemming schem Gemisch, Färbung mit Eisenhămatoxylin) habe ich die Cytomikrosomen von v. la Valette St. George (die Mitochondrien Bendas) in den Spermatocyten von Schmetterlingen schon zu einer Zeit dargestellt, bevor noch Bendas erste, den Gegenstand betreffende Publikationen erfolgt waren, in denen er übrigens über die von ihm benutzte Methodik zunächst nichts bekannt gab (vergl. auch Meves 1900, S. 554).

Ich kann nicht finden, dass die Tinktion mit Eisenhämatoxylin der später von Benda empfohlenen mit Krystallviolett nachsteht, so sehr die letztere durch die von Bend a getroffenen Verbesserungen, welche Duesberg und ich (1907) haben mitteilen dürfen, an Sicherheit gewonnen hat. Die letztere Farbung gelingt übrigens an Material, welches mit Fle m ming schem Gemisch (von der oben angegebenen Zusammensetzung) fixiert ist, auch dann, wenn die von Benda vorgeschlagene Nachbehandlung der fixierten Objekte mit einer Mischung von Holzessig und $1 \%$ iger Chromsäure und mit einer $2 \%$ igen Lösung von Kaliumbichromat unterblieben ist.

Schliesslich dürften auch noch die "Methoden der Granulauntersuchung" zur Anwendung kommen können, welche Altmann (1890) empfohlen hat, dessen „Körner und Fäden" jedenfalls vielfach mit Mitochondrien und Chondriokonten identisch sind.

b) Darstellung der Befunde.

Die Zellen von Hühnerkeimen zwischen der 15, und 22. Stunde der Bebrütung enthalten neben zahlreichen Dotterkügelchen selbstăndige gewundene Fäden, Chondriokonten, die durch besondere Feinheit ausgezeichnet sind. Fig. 1 (von einem Embryo von ca. 20 Stunden) zeigt ein Stück eines Querschnitts seitlich von der Primitivrinne. In den Zylinderzellen des Ektoderms (ak) sind die Chondriokonten lang und verlaufen parallel der Längsachse der Zelle. Kürzere Fäden ohne bestimmte Anordnung weisen die Zellen des mittleren und inneren Keimblatts (mk und ik) auf. Vielfach sieht man hier Punkte, welche sich beim Heben und Senken des Tubus als Fadendurchschnitte erweisen.

Im Beginn des zweiten Tages der Bebrütung tritt eine Änderung im Aussehen dieser Gebilde ein.

Man vergleiche die Fig. 2-17 und 27-30, welche samtlich von demselben Embryo mit acht Urwirbeln von der ca. 27. Stunde der Bebrütung stammen. 
Fig. 27-30 sind bei schwăcherer Vergrösserung gezeichnet. Fig. 27 gibt einen Querschnitt durch den hintersten Teil des Kopfes, hinter dem Eingang in die Kopfdarmhöhle, wieder. Fig. $28 \mathrm{a}$ und $\mathrm{b}$ ist ein durch das hintere Ende der Primitivrinne hindurchgelegter Querschnitt durch die Area pellucida und den inneren Teil der Area opaca. Das rechte Ende der Fig. 28 a setzt sich mit dem linken der Fig. $28 \mathrm{~b}$ fort. Fig. 29 stellt einen Querschnitt durch einen Urwirbel, Fig. 30 einen solchen durch den vorderen Teil der Herzanlage dar.

Die Fig. $2-17$ von demselben Embryo, welche bei dreimal so starker Vergrösserung gezeichnet sind, betreffen einzelne Zellen oder Gruppen von solchen.

Die eingetretenen Verïnderungen sind folgende: Die Chondriokonten haben in einem Teil der Zellen die Fadenform bewahrt, in anderen dagegen sind sie stabförmig geworden; in allen Zellen aber sind sie gegenüber Fig. 1 deutlich verdickt. An Stelle von Fäden oder Stäben findet man vielfach auch Kugeln oder Körner, Mitochondrien, welche in verschiedenen Zellen ein verschiedenes Kaliber besitzen (man vergleiche Fig. 9, 11, 12, 17).

Die dickeren Fäden, Stäbe und Körner könnten aus den langen dünnen Füden der Fig. 1 durch Verkürzung und Verdickung entstanden sein.

Es gibt aber noch eine andere Möglichkeit der Entstehung: Die kurzen dicken Stäbe und die grösseren Körner lassen eine mit Eisenhämatoxylin schwarz färbbare Schale und einen hellen Inhalt unterscheiden. An den kleineren Körnern und den dïnneren Stäben und den Făden dagegen ist ein delartiger Bau nicht erkennbar; was nicht ausschliesst, dass er auch hier vorhanden ist. Es wăre daher denkbar, dass das Dickerwerden der Fäden im wesentlichen auf einer Hohlraumbildung im Innern beruht (welche möglicherweise mit einer Substanzauflockerung einhergeht); die Stäbe und Körner könnten durch Querteilung von langen Fäden entstanden sein. Eine Zusammensetzung aus zwei Substanzen habe ich schon früher (1900) an den Mitochondrien in den Spermatocyten von Pygaera aufgefunden; auch die Chondriokonten in sich teilenden Spermatocyten der Honigbiene sind nach meiner Beschreibung (1907, 1, S. 428) nicht solide, sondern stellen Röhren dar, deren Wandung intensiv färbbar ist. 
Die etwas längeren Stäbe und die Fäden sind durchaus nicht immer gerade, sondern meistens mehr oder weniger stark gebogen, gewunden oder geknickt. Sie bängen niemals untereinander zusammen, sondern sind ganz selbständige "wohlindividualisierte" Gebilde. Häufig ziehen sie sich kreuzend übereinander hinweg, wodurch Zusammenhänge zwischen ihnen vorgetäuscht werden können.

In langgestreckten Zellen, wie z. B. in hohen Epidermiszellen, in den Zellen des Medullarrohrs, in den Zellen des Vorderdarms, des myoepikardialen Mantels sind die Fäden oder Stäbe der Hauptsache nach parallel der Längsachse der Zelle orientiert. Diese Anordnung ist um so ausgesprochener, je schmäler und je stärker in die Länge gestreckt die Zellen sind. Bei allen anderen Zellformen, in kubischen, platten, rundlichen, verästelten Zellen, zeigen die Fäden keine bestimmte Anordnung.

Auf die drei Keimblätter verteilen sich die Fäden und Stäbe bezw. Körner folgendermassen:

Die Zellen des Ektoderms enthalten grösstenteils Faden oder Stäbe und nur ausnahmsweise Körner. Man vergleiche ausser Fig. 27 und 28: Fig. 2, eine Gruppe von drei Epidermiszellen mit stäbchenförmigen Chondriokonten, welche in der mittleren der drei Zellen am dicksten sind; Fig. $3-6$ vier weitere Epidermiszellen, einzeln, in Mitose; die drei ersten (Fig. 3-5) enthalten Fäden, die letzte (Fig. 6) ziemlich voluminöse Körner; Fig. 7 ein Stiick aus der Wand des Medullarrohrs; Fig. 8, die obere Zellschicht: platte Ektodermzellen aus dem Bereich der Area opaca, welche kurze wellige Fäden einschliessen (darunter Hautfaserblatt).

Auch die Zellen des Entoderms weisen vorwiegend Fäden auf. Man betrachte die platten Entodermzellen, wie sie sich in Fig. 27 unterhalb des Medullarrohrs und in Fig. $28 \mathrm{a}$ im Bereich der Area pellucida finden; ferner die hohen Zellen, welche in Fig. 30 die untere Wand der geschlossenen und in Fig. 27 die seitlichen Wände der noch offenen Kopfdarmhöhle begrenzen. Ein abweichendes Bild bieten die hohen Zellen des Entoderms, welche in der Peripherie des hellen Fruchthofs gelegen sind, und die grossen, ungemein dotterreichen Zellen des "Keimwulstes“, welche sich an die ersteren peripheriewärts anschliessen. Diese beherbergen neben den Dotterkugeln zahlreiche feinste Körnchen (zum Teil auch Fädchen), sodass sie wie bestäubt aussehen: man 
vergleiche Fig. 28 b; eine einzelne Zelle, welche relativ wenigr Dotterkugeln einschliesst, aus der Peripherie des hellen Fruchthofes, ist in Fig. 17 gezeichnet.

Von den Zellen des mittleren Keimblatts enthalten diejenigen des Mesenchyms Körner oder kurze Stäbe (Fig. 9, 11, 12, 27, 28, 30), nur ausnahmsweise Faden (Fig. 10); ebenso die Urwirbelzellen (Fig. 29). Längere Fảden finden sich in den Zellen, welche das Coelom begrenzen; in den Herzwandzellen (Fig. 13 und 30), welche, wie Fig. 13 zeigt, schon bei einem Embryo von acht Urwirbeln durch Querbrücken zusammenhängen; ferner in den Zellen der Blutinseln (Fig. $1 \neq$ und $2 S$ ); kürzere in den jungen Blutzellen (Fig. 15 und 16), welche nach dem Hohlwerden der Blutinseln im Innern der Gefassanlagen, dichte Haufen bildend. zusammenliegen.

Bei Hiilhnerembryonen des dritten und vom Beginn des vierten Tages finde ich in allen Zellen der Organe und Systeme. die aus den drei Keimblattern hervorgegangen sind, Fäden von annahernd dem gleichen Kaliber.

Man werfe zunicbst einen Blick auf die Fig. :2, von einem Embryo von ca. 50 stunden, Teil eines Schnittes durch die Augenanlage, welcher Chondriokonten in den silmtlichen Zellen der Cornea, der Linse, der sekundaren Augenblase zeigt.

Fig. $18-26$ und Fig. 31 und 33 betreffen einen Embryo vom Beginn der zweiten Hälfte des dritten Tages (genaues Alter: 2 Tage 15 Stunden).

Fig. 31 stellt einen Teil eines durch die Rückengegend gelegten Schnittes dar.

Das Mark besteht auf diesem Stadium belianntlich aus lang spindelförmigen Zellen, welche mit ihren Auslaufern bis an die innere und aussere OberAlache heranreichen. IDiese, die sog. Radiarzellen, schliessen sehr lange Chondriokonten ein. Ebensolche, aber anscheinend kürzere, Faden umgeben in den sog. Keimzellen in unregelmåssiger Anordnung die Teilungsfigur. Nebenbei sei bemerkt, dass ich die Keimzellen nicht als eine besondere Zellart ansehe, sondern (wie auch eine Anzahl anderer Autoren) als Radiärzellen, welche sich im Beginn der Mitose abgekugelt haben. Fadchen.

Die Spinalganglienzellen enthalten in Fig. 31 kurze wellige 
Die Zellen des Urwirbels, welche um die 27. Stunde der Bebrütung vorwiegend Körner oder kurze, gerade Stäbchen einschlossen, beherbergen jetzt längere Fäden von welligem oder geknicktem Yerlauf. Eine Zellgruppe aus dem Urwirbelkern ist in Fig. 26 bei starker Vergrösserung gezeichnet.

Die zylindrischen Zellen des Cutisblattes entsenden gegen die Epidermis Fortsätze, in welche sich Chondriokonten hineinerstrecken. Die Fortsätze spitzen sich zu und laufen in einen oder mehrere dünne Fäden aus. Diese verflechten sich und stellen ein zierliches Gerüstwerk zwischen Cutisblatt und Epidermis her. Die Fortsätze selbst kommen folgendermassen zustande: Entweder ist die ganze dem Ektoderm zugekehrte Fläche einex Cutisblattzelle zungenförmig verlängert, oder die Mitte dieser Fläche erbebt sich zu einem spitz auslaufenden Kegel ${ }^{1}$ ); oder aber man sieht auf einem Querschnitt wie Fig. 31 eine der beiden seitlichen Ecken einer Endfläche (mitunter auch beide) in eine mehr oder weniger lange Spitze ausgezogen, welche mit der Längsachse der Zelle einen Winkel (bis zu annähernd $90^{\circ}$ ) bildet. Ähnliche Fortsätze gehen auch von denjenigen Zellen aus, welche die mediale, dem Medullarrohr zugewandte Seite des Urwirbels bilden.

Ein Stück Corium- und Muskelblatt eines weiter nach vorn gelegenen Urwirbels mit Epidermis darüber (von demselben Embryo) ist in Fig. 25 bei starker Vergrösserung wiedergegeben. Die Zellelemente des Muskelblatts (die primitiven Muskelfasern) sind der Quere nach getroffen; ebenso die in ihnen enthaltenen Chondriokonten, welche die Vorläufer der Myofibrillen darstellen.

Von demselben Embryo stammen ferner noch: Fig. 33, ein Schnitt durch die Darmwand; Fig. 24, Gruppe von Darmepithelzellen, bei starker Vergrösserung gezeichnet; und schliesslich, Fig. 18-23, ebenfalls stark vergrössert, eine Anzahl roter Blutzellen, Fig. 18-20 in Flachenansicht, Fig. 21 in Kantenansicht, Fig. 22 und 23 in Teilung begriffen; die Blutzellen sind durch den Besitz von auffallend langen Chondriokonten ausgezeichnet.

1) Die kegelförmigen Fortsätze gleichen den von v. Lenhossék (Die Entwicklung des Glaskörpers, Leipzig 1903) an den Linsenzellen von Säugetierembryonen beschriebenen „Basalkegeln", in welche sich ebenfalls Chondriokonten hineinerstrecken, wie mir Schnitte durch das Auge eines wahrscheinlich 27 Tage alten Hundeembryos zeigen. 
Die Schnitte, welche den Figuren $34-37$ zugrunde liegen, gehören einem Embryo von der ersten Hälfte des vierten Tages an (das genaue Alter beträgt 81 Stunden oder drei Tage und 9 Stunden).

Fig. 34 ist ein Schnitt durch die Wandung des Herzohrs. Diese bietet das Bild einer einheitlichen, von hellen Ratumen durchsetzten Protoplasmamasse, innerhalb deren Zellgrenzen nicht oder nur undeutlich zu erkennen sind. Das Protoplasma enthalt lange, stark gewundene Chondriokonten, welche stellenweise, besonders in der Nachbarschaft der Kerne, zu dichteren Knäneln zusammengeballt sind. Die Chondriokonten strecken sich später gerade und differenzieren sich zu Myofibrillen.

Fig. 35 ist ein Querschnitt durch den dorsalen Teil einer Rückenmarkshälfte, in welcher eine Anzahl Neuroblasten sichtbar sind, deren Achsenzylinderfortsätze sich ventralwärts erstrecken. Die Neuroblasten enthalten die gleichen Chondriokonten bezw. Ketten von solchen wie die Spongioblasten. Dieses Bild betrachte ich als beweisend dafür, dass die Chondriokontenketten der Neuroblasten die primitiven Neurofibrillen darstellen. Denn es sind offenbar die gleichen Fäden, welche sich bei der Silberimprägnation nach $R$ amón y Cajal schwarz färben.

Die fertigen Neurofibrillen (auf späteren Stadien der Ontogenese) sind durch die Mitochondriamethoden nicht mehr fürbbar; ebensowenig ist es bisher geglückt, die Vorlüufer der Neurofibrillen in den Zellen des Medullarrohrs ror dem Beginn des dritten Tages durch Silberimpragnation darzustellen. Besta (1904) und Held (1905) ist dieses erst von der 60., Ramón y Cajal (1908) von der 52. Stunde der Bebrïtung an gelungen. Mit diesem Zeitpunkt beginnt ein Abschnitt der Entwicklung, während dessen die Neurofibrillen sowohl durch die Mitochondriamethoden, als auch durch Silberimprägnation darstellbar sind.

In Fig. 36 habe ich ein Spinalganglion (mit umgebendem Mesenchymgewebe) und ein Stück Rückenmark mit der austretenden vorderen Nervenwurzel abgebildet. Einzelne der Spinalganglienzellen lassen zwei Fortsätze, einen zentralen und einen peripheren, erkennen, welche Chondriokonten bezw. Bündel von solchen entbalten. Die Erörterung sonstiger in Fig. 36 sichtbarer Details verschiebe ich auf eine spätere Arbeit. 
Die primitiven Neurofibrillen in sämtlichen Zellen des Medullarrohrs sind übrigens bereits 1890 von Altmann bei einem Katzenembryo, welcher sich „auf jener Entwicklungsstufe befand, in welcher die vorderen Wurzeln gerade deutlich angelegt waren", nach Fixierung mit Kaliumbichromat-Osmiumsäure durch differenzierte Färbung mit Säurefuchsin-Pikrinsäure dargestellt worden. Altmann gibt in den Fig. 1 und 2 seiner Taf. XIV Querschnitte durch ein Stück Hirnwand und durch ein Stück Vedullarrohr an der Stelle der vorderen Wurzel. Die hier sichtbaren Fibrillen sind nach Altmann (S. 53) "aus hintereinander aufgereihten Granulis zusammengesetzt". Ich bemerke beiläufig, dass ich selbst ähnliche Bilder bei Mäuseembryonen erlıalten habe, bei denen die primitiven Neurofibrillen aus Ketten von Körnchen und kurzen Fadenstücken bestehen, während sie bei Meerschweinchenembryonen ebenso wie bei denen des Huhns aus längeren Fadenstücken gebildet werden.

Die Fig. 37, Hälfte eines Querschnitts durch die Chorda, bietet besonders dann Interesse, wenn man sie mit der Chorda der Fig. 31 vergleicht. Während nämlich in Fig. 31 (von einem Embryo von 63 Stunden) die Chordazellen gewundene Fäden enthalten, schliessen sie in Fig. $3 T$ (von einem Embryo von 81 Stunden) Körner ein. Es zeigt sich also, dass die Erscheinungsform der Mitochondriensubstanz in einer und derselben Zellenart auch später noch innerhalb kurzer Zeit wechseln kann.

Zum Schluss mögen noch einige Bemerkungen über das Verhalten der Chondriosomen bei der Teilung der embryonalen Zellen Platz finden.

Fast in sämtlichen, bei schwacher Vergrösserung gezeichneten Figuren der Taf. XL-XXLII finden sich mehr oder weniger zahlreiche sich teilende Zellen; ebensolche sind auf Taf. XXXIX bei starker Vergrösserung in Fig. 1 (obere Zelle links), Fig. 3-6, $11-12,22$ und 23 abgebildet.

In den Spermatocyten erster Ordnung wirbelloser Tiere ordnen sich die hier vorhandenen Mitochondrien im Beginn der Teilung bekanntlich vielfach zu Fäden zusammen. Diese lagern sich meistens früher oder später parallel der Spindelachse, sodass sie die Teilungsfigur mantelförmig umgeben. Andere Nale bleiben sie auf einer Seite derselben liegen. 
In den Zellen des Hühnerembryos dagegen scheint der Ablauf einer Mitose auf das Verhalten und die Lagerung der Mitochondrien und Chondriokonten ganzlich obne Einfluss zu sein. Die Mitochondrien erhalten sich während der Teilung als solche ${ }^{1}$ ) und nehmen keine besondere Anordnung an; ebenso bleiben auch die Chondriokonten unregelmässig durch den Zellleib verteilt.

\section{Die Chondriosomen \\ in ihrem Verhältnis zur Filarmasse Flemmings. Über Protoplasmastruktur.}

Nachdem ich das Vorhandensein der Chondriokonten in den embryonalen Zellen konstatiert hatte, handelte es sich für mich zunăchst einmal darum, Klarheit über ihre Beziehung zur Filarmasse Flemmings zu bekommen. Dieselbe Frage hatte sich Be n d a $(1899,1)$ bereits mit Bezug auf die Mitochondrien vorgelegt und sie dahin beantwortet, dass die Mitochondrien teils deutlich den Plasmafäden eingefügt sind, teils durch ihre Anordnung ihre Zugehörigkeit $z u$ diesen erkennen lassen. Die Mitochondrien, sagte er, sind ein wohlcharakterisierter Bestandteil eines beschränkten Teiles der Faden; sie geben das Baumaterial zu einem grossen Teil bekannter intracellulärer Faden- und Faserstrukturen.

Ich habe demgegenüber 1900 (S. 598) für meine damaligen Untersuchungsobjekte (Samenzellen von Paludina und Pygaera) betont, dass die Mitochondrien wăhrend der Mitose sicher in terfilar, sowohl ausserhalb der Spindelfasern (was auch Benda bereits festgestellt hatte) als auch ausserhalb der Polstrahlungen liegen. Ich konnte daher auch Benda nicht beistimmen, wenn er in den innerhalb der Polstrahlen gelegenen "Mikrosomen Van Benedens, M. Heidenhains und v. Kostaneckis" Mitochondrien wieder zu finden glaubte.

Dagegen meinte ich 1900 bei Pygaera konstatieren $\mathrm{zu}$ können, dass die Mitochondrien der ruhenden Samenzelle untereinander durch schwach färbbare Fäden verbunden sind, welche in zwei oder mehr Richtungen von ihnen abgehen, dass sie also hier in der Tat eine intrafilare Lage haben, fand aber bei erneuter

2) Ebenso nach meinen Beobachtungen (1900, S. 560) in denjenigen Spermatocyten von Paludina, welche oligopyrene Spermien liefern, und nach Duesberg $(1907$, S. 290) in den Spermatocyten der Ratte. 
Prüfung $(1907,2)$ doch, dass die bezüglichen Beobachtungen unsicher seien. Bei den ruhenden Zellen junger Embryonen vermochte ich über den in Rede stehenden Punkt nichts auszumachen, weil eine Filarmasse neben den Mitochondrien oder Chondriokonten an meinen Präparaten überhaupt nicht erkennbar war; jedoch fand ich es wenig wahrscheinlich, dass die zum Teil sehr langen Chondriokonten der embryonalen Zellen noch wieder in Plasmafäden eingelagert seien.

Um in dieser Frage weiter zu kommen, habe ich $(1907,3)$ die Methoden der Mitochondriadarstellung auf diejenigen Objekte angewandt, auf welche Flemming seine Fadenbaulehre gegründet hat.

Dabei machte ich nun die Entdeckung, dass die von Flemming in lebenden Zellen der Salamanderlarve beobachteten Faden sich in der Farbe der Mitochondrien intensiv tingieren. Es handelt sich demnach um Chondriokonten, oder, mit anderen Worten, die Faden, welche ich als Chondriokonten in den Zellen junger Embryonen beschrieben habe, sind Filarmasse im Sinne Flemmings.

Man wolle sich erinnern, das Flemming 1882 von seiner Filarmasse folgende Schilderung gab. Im Zellenleib lassen sich ausser dem Kern und etwaigen besonderen Körnereinschlüssen zwei verschiedene Substanzen unterscheiden, von denen die eine etwas stärker lichtbrechend und in Form von Fadenwerken angeordnet ist, während die andere den bleibenden Raum ausfüllt. Flemming weicht von seinen Vor- und Mitarbeitern darin ab, dass er kein Recht findet, die Fadenwerke ohne weiteres netzförmig zu nennen. Wenn er auch eine netzförmige Beschaffenheit für viele Objekte als völlig möglich zugibt, kann er doch keine Sicherheit dafür finden. Auch an den Zellenarten, welche die Fadenwerke besonders scharf und klar zu sehen erlauben, liegt die Frage (selbst für die besten Linsen) noch an der Grenze des Sichtbaren, ob die Fäden sich wirklich gerüstförmig verbinden, ob sie vielfach oder gar durchweg nur aneinander vorbeiaufen, oder endlich, ob das Fadenwerk unterbrochen ist, nur aus einzelnen, gleichmăssig gelagerten Stücken besteht.

Flemming ist es nicht oder nur teilweise geglückt, die von ihm am lebenden Objekt gesehenen Strukturen naturgetreu zu fixieren; jedenfalls aber hat er sie auch am fixierten Präparat nur in ungefärbtem Zustand vor sich gehabt. Um so grössere Bewunde- 
rung verdient die Schärfe seiner Beobachtung, die es ihm ermöglichte, Beschreibungen davon zu geben, denen sich auch auf Grund des studiums fixierter Präparate, an welchen die Fitden durch intensive Farbung hervorgehoben sind, kaum etwas hinzu-

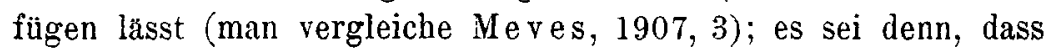
sich am gefärbten Objekt in beinahe allen Fallen mit Bestimmtheit konstatieren lasst, dass die Fuden in den von Flemming studierten Zellen der Salamanderlarve nicht miteinander zusammenhängen, sondern in der Tat, wie Fl e m m ing damals anzunehmen geneigt war, selbständig sind.

Damit soll aber nicht bestritten werden, dass bei bestimmten Objekten netzartige Anordnungen der Fila oder Chondriokonten vorkommen. Ich selbst habe erst kürzlich $(1907,1)$ beschrieben, dass die Chondriokonten in den Spermatocyten der Honigbiene auf einem bestimmten Stadium in Form eines des Kern umgebenden Netzwerks angeordnet sind. Ein weiteres Netzwerk, dessen Balken allem Anschein nach ebenfalls von Chondriokonten gebildet werden, findet sich an der Oberfläche der Leydigschen Zellen in der Epidermis der Salamanderlarve (vergl. Meves, 1907, 3). Hierher gehören möglicherweise auch die „Netzapparate“, welche neuerdings im Cytoplasma sehr verschiedener Zellarten von Golgi (zuerst 1898), Negri (1899), Ballowitz (1900), Pensa (1901), Kopsch (1902), v. Bergen (1904) und anderen beschrieben worden sind. Mit Bezug auf das Binnennetz der Ganglienzellen sind Popoff (1906) und Van Durme (1907) bereits zu dem Resultat gekommen, dass es dem „Mitochondrialapparat" der Geschlechtszellen homolog ist.

Die Feststellung, dass die Fle $\mathrm{m} \mathrm{m}$ in $\mathrm{g}$ schen Fila mit Chondriokonten identisch sind, ist geeignet, eine höchst erwünschte Klärung in unseren Anschauungen über die Struktur der Zellsubstanz herbeizufübren.

Diejenige Substanz, welche die Fäden oder Chondriokonten bildet, kann, wie wir nunmehr wissen, auch in Form von Körnern vorkommen. Diese Körner, die Mitochondrien, verlieren damit die Sonderstellung, die ihnen bisher zuerkannt werden musste; sie sind nur eine andere Erscheinungsform der Fle m mingschen Fila.

Nach meiner Meinung lässt sich mit Sicherheit behaupten, dass ein grosser Teil der von Altmann beschriebenen Granula (wahr- 
scheinlich fast alle, soweit sie nicht Reagentienprodukte sind) mit Mitochondrien identisch sind. Meine Befunde sind demnach geeignet, die Granulalehre Altmanns und die Fadenbaulehre Flemmings miteinander zu versöhnen, insofern sie zeigen, dass es sich bei dem Fadenbau der Zellsubstanz nicht um ein allgemeines Prinzip handelt, dass die Anordnung in Fäden durchaus nicht (Waldeyer, 1895, S. 847) "das Wesen der Struktur bildet". Jedoch ist Flem ming im Recht, wenn er darauf besteht (zuletzt 1899, S. 9), dass die von ihm beobachteten Fäden kontinuierliche Stränge und nicht Aufreihungen von Körnern darstellen.

Dass auch bei pflanzlichen Zellen Chondriokonten vorkommen, ergab sich aus Beobachtungen, welche ich (1904) zuerst bei den Tapetenzellen von Nymphaea gemacht babe und welche Tischler (1906) bei denjenigen von Ribes bestätigt hat. Die Chondriokonten liegen hier in einer Zellsubstanz, welche, wie bei den meisten pflanzlichen Zellen, dicht von Hiüssigkeitshaltigen Hohlraumen, Vakuolen, durchsetzt ist. Strukturen, die mit den von Tischler und mir beschriebenen identisch sind, wurden ferner von v. Smirnow (1906) in den Zellen von Hyazinthenwurzeln und Erbsenkeimen, sowohl in frischem als auch in fixiertem und gefärbtem Zustand, wahrgenommen. Über weitere Chondriosomenbefunde bei pflanzlichen Zellen wird demnächst Lams berichten.

Nachdem sich nunmebr herausgestellt hat, dass die Chondriokonten mit den Fila Flemmings identisch sind und dass an Stelle der Fila auch Körner ("Mikrosomen", "Grana") vorhanden sein können, kommen wir auch auf botanischem Gebiet mit einer grösseren, teilweise älteren Literatur (Strasburger [1884, 2], Pfeffer [1886], Bertbold [1886], E. Zacharias [1888], welcher die bezüglichen Ergebnisse seiner Vorgänger resumiert, Mikosch [1896], vielleicht auch Němec [1901] und andere) in Berührung; ein Eingehen auf dieselbe würde an dieser Stelle zu. weit führen.

Ob auch die Chromidien der Protozoen, wie von mehreren Seiten angenommen wird, tatsächlich mit Mitochondrien identisch sind, darüber kann ich mir ein Urteil nicht erlauben, um so weniger, als anscheinend ganz heterogene Dinge in den Protozoen unter diesem Namen beschrieben worden sind (vergl. Goldschmidt, 1904, S. 69). - 
Diejenige Substanz, welche die Filarmasse durchlagert, die sogen. Interfilarmasse, bezeichnete Flemming 1882 als („anscbeinend wenigstens") byalin oder homogen. Er gibt es aber als möglich $\mathrm{zu}$, dass sie noch wieder eine feinfädige Beschaffenheit als präformierte Struktur in sich besitzen könnte. Die „feinen Fadenwerke", welche an fixierten Präparaten sichtbar sind, könnten jedoch auch weiter nichts als Kunstprodukte sein, die durch die Reagentien neben den bereits in der lebenden Zelle vorhandenen Fäden und Körnern (Chandriosomen) neu erzeugt worden sind.

In der 'lat halte ich es für sehr wahrscheinlich, dass in vielen embryonalen Zellen alle Plasmastruktur des Ruhezustandes durch Chondriosomen repräsentiert wird.

Nun gibt es aber unzweifelhaft Fadenstrukturen, die weder Chondriokonten sind, noch auch, soviel wir bisher wissen, in irgend einer genetischen Beziehung zu den Chondriosomen stehen; Fadenstrukturen, die zwar vital vielfach unsichtbar, aber doch sicher keine Artefakte sind: das sind die Strahlungen, welche allgemein in sich teilenden Zellen, mitunter auch schon wahrend des Ruhezustandes, von den Cytocentren ausgehen. Auf Grund ihrer Existenz erscheint es annehmbar, dass statt ihrer in solchen Zellen, in denen sie wăhrend des Ruhezustandes fehlen, gleich oder äbnlich beschaffene Fadenwerke in anderer, z. B. netz- oder gerüstförmiger Anordnung intra vitam existieren könnten. Als solche könnten eben die erwähnten "feinen Fadenwerke" in Betracht kommen, welche in vielen fixierten Prăparaten neben den vergleichsweise gröberen Faserungen vorbanden sind, vorausgesetzt eben, dass sie nicht durch die Reagentien ausgefällt worden sind.

Sehen wir von den letzteren ab und bleiben wir bei den Strahlungen, deren Vitalität nicht $\mathrm{zu}$ bezweifeln ist, so können demnach jedenfalls zwei verschiedene Fadenaiten in der Zellsubstanz nebeneinander vorkommen. Flem ming hat die "Protoplasmastrahlungen ", z. B. diejenigen in den Leukocyten, auf eine Stufe mit den Fila gestellt, welche er 1882 in den lebenden Salamanderzellen beobachtet hat. Diese Homologisierung ist, wie sich nunmehr herausgestellt hat, nicht berechtigt. Im Cytoplasma des Leukocyten liegen Chondriokonten und Mitochondrien zw is chen den Plasmastrahlen, welche von den Centriolen ausgehen (vergl. Me ves, 1907, 3). 
Die Entwicklung, welche unsere Kenntnisse der Plasmastruktur in den letzten Jahren genommen haben, lisst es vielleicht gerechtfertigt erscheinen, wenn wir die Flemmingschen Bezeichnungen Filarmasse oder Mitom auf die Strahlungen und die bezüglich ihrer vitalen Existenz noch zweifelhaften, ihnen eventl. gleichwertigen feinen Faden- oder Netzwerke beschränken. Dagegen könnte die Gesamtheit der in einer embryonalen Zelle vorhandenen Chondriosomen als Chondriom bezeichnet werden.

Man kann übrigens die Vermutung haben, dass zwischen dem Chondriom einerseits und der Filarmasse oder dem Mitom im obigen Sinne andererseits Wechselbeziehungen vorhanden sind in der Weise, dass das Chondriom sich in Filarmasse und eventuell umgekehrt umwandelt.

\section{Die Rolle der Chondriosomen bei der Zell- differenzierung und Vererbung.}

Die im vorigen Kapitel gegebene Darstellung der Zellstruktur gilt im wesentlichen für embryonale Zellen, solange sie sich in einem indifferenten Zustand befinden.

Mit der Sonderung des Embryonalleibs in verschiedene Organe und Gewebe übernehmen die zuerst gleichartigen Zellen besondere Funktionen, die in besonderen Strukturen oder Differenzierungen ihren Ausdruck finden.

Alle diese Differenzierungen, so heterogen sie sind, entstehen nun durch Metamorphose eines und desselben elementaren Plasmabestandteiles, der Chondriosomen. Die Chondriosomen sind das den Differenzierungsprozessen zugrunde liegende materielle Substrat, welches , in den spezifischen Substanzen der verschiedenen Gewebe different" wird.

Das Studium eines Teils dieser Umwandlungen soll das Thema weiterer Arbeiten bilden; hier sei darüber nur kurz folgendes bemerkt.

$\mathrm{Zu}$ den Differenzierungsprodukten der Chondriosomen gehören zunächst einmal die verschiedensten Faserstrukturen : zahlreiche fibrilläre Bildungen in Epithelzellen, z. B. nach meinen demnächst zu beschreibenden Beobachtungen die „Protoplasmafasern “ der Epidermiszellen, ferner die Fibrillen der glatten und 
quergestreiften Muskelfasern, ${ }^{1}$ ) die Neurofibrillen und Neurogliafasern, die Bindegewebsfasern. ${ }^{2}$ )

Man kann unter diesen Fasern mitochondrialer Herkunft die beiden Gruppen der gegliederten und ungegliederten Fasern unterscheiden.

Als gegliederte Fasern bezeichne ich die „Körnerfäden“ oder die Chondriomiten Bendas, das sind Reilien von Mitochondrien, die durch Zwischenglieder verknüpft werden, welche durch die Mitochondrienmethoden nicht farbbar sind. Dahin gehören z. B.: die Wimperwurzeln in Flimmerzellen von Helix (nacb Benda), die sog. Kopulationsfäden der Sertolischen Zellen (ebenfalls nach Benda), ferner die quergestreiften Muskelfibrillen. Chondriomiten können dadurch entstehen, dass Mitochondrien sich in Reiben anordnen und durch nicht färbbare Zwischenglieder in Verbindung treten, oder aber dadurch, dass Chondriokonten sich in Fäden differenzieren, welche abwechselnd aus zwei verschiedenen Substanzarten bestehen, von denen die eine die färberischen Eigenschaften der Chondriosomen beibehält.

Andere Faserstrukturen von gleichfalls mitochondrialer Abkunft bestehen dagegen aus einer anscheinend homogenen Masse, weiche durch die zur Darstellung der Chondriosomen geeigneten Methoden nicht furbbar ist; diese Faserstrukturen lassen sich den. ersteren als ungegliederte gegenüberstellen. Hierher rechne ich z. B. die Neurotibrillen und die Fibrillen der glatten Muskelfasern.

Die Differenzierung ergreift übrigens, wie ich $z$. B. bei den Spinalganglienzellen des Hühnerembryos habe konstatieren können, keineswegs immer das gesamte Chondriom; wenn ich die Fig. 2-4 auf Taf. XXIX von v. Bergen (1904) betrachte, ist mir sehr wahrscheinlich, dass der Netzapparat dieser Zellen beim er-

1) Eine Entstehung der quergestreiften Muskelfibrillen aus Granulis bez. Mitochondrien haben bereits Altmann (zweite Aufl., 1894, S. 60) und Benda $(1899,1)$ beschrieben.

2) Flemming ist bekanntlich bereits vor längerer Zeit (1891 und 1897) zu dem Resultat gekommen, dass die Bindegewebsfasern aus dem Protoplasma dureh eine Umprägung der Fadenstruktur desselben entstehen. Ich schliesse mich dieser Ansicht anf Grund meiner eigenen Beobachtungen an, halte aber die Annahme, welche auch Flemming (1897, S. 186-187) als berechtigt anerkennt, für unbedingt notwendig: dass die zunächst cellullär gebildeten Fibrillen sich später selbständig vermehren und weiter wachsen. 
wachsenen Tier aus undifferenziert gebliebenen Chondriokonten besteht (vergl. auch Pop off und Van Durme).

Eine zweite Gruppe von Differenzierungsprodukten der Chondriosomen dürfte sich aus den verschiedensten auffälligen $\mathrm{ch} \mathrm{e} \mathrm{mi}$ $\mathrm{sc}$ hen Erzeugnissen des cellulären Stoffwechsels zusammensetzen. Altmann (1890) und Arnold (1905, 1 und 2) kaben gezeigt, dass es sich bei der Sekretion um die Umwandlung von Zellgranulis zu Sekretkörnern handelt. Die Sekretkörner nehmen den dem Drüsenlumen zugekehrten Teil der Zelle ein, während der basale Teil z. B. in den Zellen der Katzenparotis (Altmann, zweite Aut. 1894, S. 125 ; Fig. 2, Taf. XXIV) Fadchen und kleine Körner enthalt, welche, „für den Nachwuchs neuer Sekretkörner zu sorgen haben"; von diesen Fadchen und Körnern glaube ich, dass sie mit Chondriokonten und Mitochondrien identisch sind (vergl. aucb Arnold, 1907, 2, S. 646). Ebenso wie die Sekretkörner der Drüsenzellen entstehen auch andere Stoffwechselprodukte durch eine Umwandlung von Granulis bezw. Mitochondrien, z. B. das Fett (Altmann, 1890, Metzner, 1890, Arnold, 1900, 1, 1905, 1): die Pigmente (Arnold, 1900, 2, 1902, 1907, 1), die Dotterkörner der Eizelle (Van der Stricht 1902-1905 und seine Schüler DHollander 1904, Lams 1904, 1907, De Somer 1905, Van Durme 1907; ferner Russo $190 \pi$, I und 2, 1908).

Auch in den Zellen des erwachsenen Körpers könnten neben den Chondriosomen bezw. ihren Differenzierungsprodukten "feine Fadenwerke" (Mitome in dem oben erörterten Sinn) existieren. Wo solche ausschliesslich an Reagentienprapparaten sichtbar sind, ist nach dem oben gesagten die Möglichkeit im Auge zu behalten, dass sie weiter nichts als Ausfällungsprodukte darstellen.

Die schon an und für sich sehr wichtige Tatsache, dass die Chondriosomen allen Differenzierungsprozessen zugrunde liegen, gewinnt ganz besondere Bedeutung für die Morphologie der Vererbung, sobald der mit Sicherbeit zu erwartende Nachweis gelingt, dass die embryonalen Chondriosomen durch die Vereinigung der männlichen und weiblichen entstehen.

O. Hertwig hat einmal gesagt (1885, S. 290): „Von einer Substanz, welche Anlagen der Eltern auf das Kind übertragen soll, werden wir annehmen müssen, dass sie sich noch in einem ursprünglichen, histologisch undifferenzierten Zustand befindet“. 
Das ist, wie wir gesehen haben, genau der Zustand der Chondriosomen, welcher uns aber nicht hindert, uns vorzustellen, dass sie eine spezifische intime Struktur besitzen, in der potentiell alle Eigentümlichkeiten der Art (genauer: des Cytoplasmas der Art) enthalten sind. Diese Struktur könnte diejenige sein, welche $\mathrm{Na}$ a e li seinem Idioplasma zuschreibt (vergl. unten).

Die verschiedenen Differenziernngsprodukte unseres Körpers sind nun nichts als Weiterbildungen dieser selben Substanz, welche in allen embryonalen Zellen vorliegt. Sobald demnach die Beteiligung der Chondriosomen bei der Befruchtung nachgewiesen ist, ergibt sich ohne weiteres, dass der Charakter dieser Differenzierungsprodukte durch Vererbung von den Eltern ber bestimmt sein muss; nicht nur der Charakter der animalen Produkte (Myofibrillen, Neurofibrillen), sondern auch derjenige der vegetativen; insofern wahrscheinlich auch die Stoffwechselprodukte durch Umwandlung von Mitochondrien entstehen, ist es uns möglich, direkter als bisher einzusehen, wie auch diese zu denjenigen Dingen gehören können, deren Beschaffenheit sich vererbt. -

Haben wir demnach in den Chondriosomen mit grösster Wahrscheinlichkeit die spezifische zu vererbende Cytoplasmastruktur zu erblicken, so werden wir weiter zu dem Schluss genötigt, dass in ihnen auch die Bedingungen fül die Differenzierungsfäbigkeit der Blastomeren enthalten sein müssen.

Die experimentellen Untersuchungen an sich furchenden Eiern haben gelehrt ${ }^{1}$ ), dass zwei verschiedene Eitypen zu unterscheiden sind, welche übrigens durch zahlreiche Übergangsglieder verbunden sind. Bei dem ersten Typus, den sogenannten Regulationseiern, zu denen z. B. die Eier der Echinodermen und Medusen gehören, scleeint das ganze Ei in allen seinen Teilen aquipotent zu sein, so dass jedes Teilstück das Vermögen besitzt, einen vollständigen, wenu auch kleineren Embryo aus sich entwickeln zu können. Im Gegensatz dazu stehen die sogenannten Mosaikeier, bei denen die verschiedenen Eiteile verschiedene Entwicklungspotenzen in sich bergen. Sie werden z. B. durch die Eier der Ctenophoren und Gastropoden repräsentiert. Hier hat sich eine Abhăngigkeit der Organbildung von gewissen in

1) Ich verweise auf die übersichtliche Darstellung von Fischel (1902), welche dem oben ausgeführten zugrunde liegt. 
der noch ungefurchten Eizelle vorhandenen organbildenden Substanzen erweisen lassen, welche eine ganz bestimmte Lagerung in der Eizelle einnehmen und durch den in regelmässiger und ganz bestimmter Weise erfolgenden Furchungsprozess auf die einzelnen Blastomeren verteilt werden

Diese organbildenden Substanzen sind nun meines Erachtens in den Chondriosomen, also "in morphologisch ausgedrückten, optisch bemerkbaren "Strukturen im Körper der Eizelle, zu suchen. ${ }^{1}$ )

Die Chondriosomen des befruchteten Eies, welche aus der Vereinigung der elterlichen hervorgegangen sind, beharren bei den Regulationseiern in den erstentstehenden Blastomeren in einem indifferenten Zustand, bleiben daher omnipotent. Bei den Mosaikeiern dagegen fangen sie gleich nach Eintritt der Befruchtung an, sich $z \mathfrak{u}$ spezifizieren, und zwar verschieden je nach ihrer Lage im Ei, sodass den Blastomeren schon auf den ersten Furchungsstadien ein qualitativ verschieden vorgebildetes Chondriosomenmaterial zufällt. Eine Blastomere, welche im Lauf der weiteren Entwicklung nur Mesodermzellen bildet, erhält eine andere Art von Chondriosomen zuerteilt als eine solche, welche Ektoderm- oder Entodermzellen entstehen lässt.

Dieses Verhalten der Chondriosomen in den Mosaikeiern entspricht einem meines Wissens besonders von Mehnert (z. B. 1898 , S. 62) betonten, allgemeinen Gesetz, nach welchem alle wichtigeren Entwicklungsvorgänge bei der Vererbung die Tendenz zur Verfrühung zeigen, d. h. das Bestreben, immer früher zu beginnen, immer mehr dem ursprünglichen Termin des Eintretens vorauszueilen.

Bei den meisten Pflanzen und vielen niederen Tieren sind bekanntlich fast alle Körperzellen imstande, ein neues Individuım aus sich hervorgehen zu lassen; diese müssen demnach hier wenigstens noch einen Rest undifferenzierter Chondriosomen entbalten, welche sich dem mikroskopischen Nachweis nicht entziehen werden.

Indem ich den Chondriosomen eine wichtige Rolle bei der Übertragung erblicher Eigenschaften zuschreibe, denke ich

1) Die häufig zu becıbachtenden verschiedenen Färbungen des Eikörpers, bei denen es sich meist nur um grössere oder geringere Durchsichtigkeit handelt, könnten weiter nichts als, ,rein lokale Koincidenzen" sein (Driesch, 1905, S. 632). 
nicht daran, ihnen die vererbende Kraft allein $z u$ vindizieren und sie dem Kern abzusprechen. Meine Meinung geht vielmehr dahin, dass die Vererbung durch Protoplasma und Kern zusammen bewirkt wird. Die Qualitaten des Kerns werden durch die Chromosomen übertragen, diejenigen des Plasmas durch die Chondriosomen.

\section{Die Chondriosomen als das materielle Substrat der $\mathrm{Na \theta gelischen} \mathrm{Idioplasmatheorie.}$}

Unser Ergebnis, dass die Chondriosomen mit grösster Wahrscheinlichkeit als Vererbungssubstanz anzusehen sind, fordert dazu auf, zu untersuchen, wie weit die Vorstellung auf sie passt, welche Naegeli sich von der ausseren Erscheinung und der Struktur seines Idioplasmas gemacht hat.

"Idioplasma" ist ein von $\mathrm{Naegeli}$ aus theoretischer Spekulation gewonnener Begriff. Die Erscheinungen, die wir von dem Idioplasma kennen, nötigen nach Naegeli zu der Annahme, dass es aus Strïngen besteht, welche wahrscheinlich ein zusammenhangendes Tetz in Cytoplasma und Kern bilden. Dieses Netz lasst sich in doppelter Art denken: ,entweder als ununterbrochene, netzförmig anastomosierende Stringe, oder als Strangstücke von begrenzter Länge, die netzförmig zusammengeordnet sind" (S. 41).

Die Idioplasmastrange setzen sich ibrerseits aus parallelen Reihen von Wicellen zusammen; sämtliche Vicellen einer Reihe sind identisch. Die spezifische Beschaffenheit des Idioplasmas wird durch die Konfiguration des Querschnitts ausgedrückt, „in welcher die ganze Ontogenie mit allen ibren Eigentümlicbkeiten als Anlage entbalten sein muss*.

Alle elementaren Charaktere eines Organismus werden nicht durch eine einzelne Micellreihe, sondern durch Bündel von solchen dargestellt; die komplexen Charaktere werden aus elementaren kombiniert. „Das Idioplasma bringt die Anlagen für verschiedene Organe in ăhnlicher W.eise zur Entfaltung, wie der Klavierspieler auf seinem Instrument die Harmonien und Disharmonien eines Musikstückes zum Ausdruck bringt. Derselbe schlăgt für jedes a und jeden anderen Ton immer wieder die namlichen Saiten an. So sind die im Idioplasma liegenden Gruppen von Vicell- 
reihen gleichsam Saiten, von denen jede eine andere elementare Erscheinung darstellt" (S. 44).

Dieser Theorie schien es bisher innerhalb des Cytoplasmas an dem geeigneten materiellen Substrat $z u$ fehlen; was verschiedene absprechende Kritiken zur Folge hatte.

Strasburger schrieb 1884, 1 (S. 118-119): „Der wichtigste Vorwurf, der sich dieser Theorie wohl machen lässt, ist der, dass sie den Tatsachen so wenig Rechnung trägt. Der Bau des Zellkerns würde allenfalls der Schilderung genügen, welche $\mathrm{Naegeli}$ von dem hypothetischen Bau des Idioplasma entwirft, keinesfalls aber derjenige des Cytoplasma".

Nach v. Koelliker (1885, S. 13) empfindet man, „bei aller Anerkennung der geistvollen Auseinandersetzungen $\mathrm{Naegelis,}$ doch am Schluss seiner Darstellungen einen gewissen Mangel insofern, als einem sozusagen nichts in den Handen bleibt, womit man weiter bauen könnte und man umsonst sich vorzustellen versucht, wo nun die idioplastische Substanz eigentlich ihren Sitz habe und wie sie an der Gestaltung sich beteilige".

Jul. Sachs (1893, S. 1202) erklärte die Darwinschen Keime und $\mathrm{Naegelis}$ Idioplasma, „das in Form unsichtbar feiner Fàden den Organismus durchzieht", für "die sonderbarsten Blüten, welche die moderne Naturforschung gezeitigt hat"; trotzdem habe jede dieser Lehren sofort ihre begeisterten Anuanger gefunden. "Der einzige Berechtigungsgrund einer Hypothese oder einer Theorie liegt darin, dass aus ihr neue Gedanken, neue Forschungswege gefunden werden; was lässt sich aber aus diesen Phantasiegebilden ableiten?"

Auch nach O. Hertwig (1906, S. 628) besteht „die schwache Seite sowohl bei Spencers Lehre von den physiologischen Einheiten, wie bei $\mathrm{Na}$ egelis Lehre vom Idioplasma darin, dass sie reine Spekulationen sind, dass sie in der vorliegenden Form keine Verbindung mit der empirischen Forschung gestatten und sie daher auch nicht beeinflussen können. Damit fehlt ihnen aber für ihre eigene weitere Entwicklung der feste Grund und Boden, welcher in den Erscheinungen der realen Wirklichkeit für alle Naturwissenschaft gegeben ist."

Im übrigen schätzt 0 . Hertwig den wissenschaftlichen Wert der Naegelischen Theorie sehr boch; er kann daher 
"zwar in diesem einen Punkt, aber sonst in keiner Beziehung dem harten und ungerechten Urteil von J u I. S a ch s beistimmen".

In den Chondriosomen scheinen mir nun Strukturverhältnisse gefunden $\mathrm{zu}$ sein, welche eine geeignete Grundlage für die $\mathrm{Naegelische}$ Theorie innerbalb des Cytoplasmas abgeben; das "Phantasiegebilde", wie Jul. Sachs das Naegelische Idioplasma genannt hat, könnte in Gestalt der Chondriosomen Realität gewonnen haben.

Die Chondriokonten des Hühnerembryos erscheinen entsprechend einem $\mathrm{Naegelischen} \mathrm{Postulat} \mathrm{als} \mathrm{„Strangstücke} \mathrm{von}$ begrenzter Länge“, welche am ersten sowie am dritten und folgenden Tage der Bebrütung in allen Zellen des embryonalen Körpers gegenwärtig sind. In vielen Zellen des Hübnerembryos innerhalb der 24.-48. Stunde und ebenso in den männlichen und weiblichen Sexualzellen zahlreicher Tiere finden wir allerdings keine Fäden oder Stäbe, sondern Kügelchen. Um auf dem Boden der $\mathrm{Na}$ egeli schen Theorie verbleiben zu können, brauchen wir aber nur anzunehmen, dass die Kügelchen kurze Fadenstücke darstellen, oder dass sie eine Polarität besitzen und dass die Micellreihen von Pol zu Pol laufen. Die Körnchen, welche in den Spermatiden der Säugetiere den Spiralfaden bilden, könnten sich mit den Polen aneinanderreiben.

Dagegen finden die Vorstellungen, welche $\mathrm{Na}$ a geli in bezug auf die spezifische Wirksamkeit des Idioplasmas entwickelt hat, in meinen Beobachtungen über das Verhalten der Chondriosomen bei der "Entfaltung der Anlagen“ keine Bestătigung.

Die Wirksamkeit des Idioplasmas beruht nach $\mathrm{N}$ a e geli darauf, dass Micellreihenbündel bezw. Komplexe von solchen in einen Zustand besonderer Erregung hineingeraten, welche „durch dynamische Einwirkung und durch Übertragung eigentümlicher Schwingungszustände bis auf eine mikroskopisch sehr geringe Entfernung die chemischen und plastischen Prozesse beherrscht" (S. 532-533). Das Idioplasma „erzeugt weicheres Ernăhrungsplasma oft in tausendfacher Menge, und mit Hilfe desselben bewirkt es die Bildung von nicht albuminartigem Baumaterial, von leimgebenden, elastischen, hornartigen, zelluloseartigen Substanzen usw., and es gibt diesem Baumaterial die gewünschte plastische Gestait" (S. 47). 
„Ort und Zeit für das Auftreten einer jeden Erscheinung hängt von dem wechselnden Erregungszustande, in dem sich das Idioplasma befindet, und von der Einwirkung, die es von der ihm angewiesenen Stelle in der Ontogenie empfängt, ab" (S. 131).

Die Beobachtung ergibt nun aber, dass die verschiedenen Differenzierungsprodukte der Zellen nicht durch Einwirkung der Chondriosomen (bezw. der den Chondriosomen innewohnenden Molekularkräfte) auf das umgebende Cytoplasma hervorgebracht werden; sie entstehen vielmehr aus den Chondriosomen selbst auf dem Wege direkter Metamorphose, welche nach den verschiedensten Richtungen vor sich geht.

Na egeli hat (S. 48) auseinandergesetzt, dass die Micellreihen der Idioplasmastränge "in ihrer Wirksamkeit behindert" sein müssen, sobald sie nicht unmittelbar an der Oberfläche des Strangquerschnitts gelegen sind. Er hält es daher für müglich, „dass die Idioplasmastrünge, um leichter auf die Umgebung einwirken zu können, nicht einen rundlichen oder ovalen, sondern mit der Zunahme der Zahl der Anlagen einen mehr und mehr gelappten Querschnitt besitzen, wodurch die Oberfläche stark vergrössert und die einzelnen Anlagen der Oberflache genahert werden. Wenn nun auch die Chondriosomen eine Wirkung wie die von Nageli angenommene bei der Bildung der Differenzierungsprodukte nicht ausüben, so besteht doch wohl zwischen ihnen und dem umgebenden Cytoplasma ein Stoffaustausch, der durch ausgiebigere Berührung zwischen beiden erleichtert werden würde. In diesem Sinne ist es vielleicht zu verstehen, dass die Chondriokonten in den Spermatocyten der Honigbiene die Form hohler Röhren aufweisen und dass auch die Mitochondrien in den Samenzellen von Pygaera stets, in den Zellen des Hühnerembryos jedenfalls vielfach hohl sind.

Was die innere Struktur der Idioplasmastränge anbelangt, so lässt Na geli sie, wie gesagt, aus einer grossen Anzahl von Micellreihen, bezw. Bündeln von solchen zusammengesetzt sein. Einen Hinweis darauf, dass den Chondriosomen ein entsprechender molekularer Bau zukommt, hat man vielleicht darin zu erblicken, dass zahlreiche Fibrillärstrukturen aus ihnen hervorgehen. Bei den Mitochondrien, soweit sie bohl sind, könnten die Micellreihen wie die Bastfasern in der ausseren Hülle einer Kokosnuss angeordnet sein. 


\section{VIII. Über die Frage, wie weit die Chondriosomen den von der Kernsubstanz in ihrer Eigenschaft als Erbmasse erfüllten Bedingungen genügen.}

Unter den Gründen, welche von 0 . Hertwig zugunsten der Annahme geltend gemacht werden, dass der Kern der alleinige Vererbungsträger sei, ist der wesentlichste der, dass das Chromatin den Forderungen, welche wir an eine "Erbmasse" zu stellen berechtigt seien, durchaus entspricht. Diese Forderungen sind: 1. dass männliche und weibliche Erbmasse sich ăquivalent gegenübersteht; 2. dass die sich vermehrende Erbmasse auf die aus dem befruchteten Ei hervorgehenden Zellen gleichwertig verteilt wird; 3 . dass eine Summierung der Erbmasse verhütet wird.

Dadurch, dass die Kernsubstanz diese Forderungen erfüllt, wird es meines Erachtens in der Tat im höchsten Grade wahrscheinlich, dass das Chromatin Erbmasse ist; aber es wird dadurch nicht bewiesen, dass "Kernmasse und Erbmasse dasselbe ist" oder dass die Kernmasse die einzig existierende Erbmasse ist.

Es entsteht nun die Frage, ob oder wie weit auch die Chondriosomen, welche ich als die cytoplasmatische Vererbungssubstanz anspreche, den gleichen Bedingungen genügen.

Dass jede der beiden kopulierenden Geschlechtszellen dem Keim gleich viel Erbmasse überliefert, ist nach 0. Hertwig ein als Wahrheit sich von selbst aufdrängender und daher gleichsam als Axiom verwertbarer Gedanke.

Demgegenüber steht wohl ohne weiteres fest, dass die Menge der in der Eizelle vorhandenen Mitochondrien sehr viel grösser ist als diejenige, welche mit dem Samenfaden eingeführt wird. Diese Tatsache bereitet uns aber keine irgendwie erheblichen Schwierigkeiten, sobald wir annehmen, dass die spezifische Beschaffenheit der Chondriosomen wie diejenige der Idioplasmastränge durch die Konfiguration des Querschnitts ausgedrückt wird. Naegeli rechnet selbst durchaus mit der Möglichkeit, dass bei der Befruchtung gewöhnlich ungleiche Mengen von Idioplasma zusammenkommen. Nach ihm würde daraus nur zu folgern sein, „dass ein allfälliger Überschuss von mănnlichem oder weiblichem Idioplasma beim Befruchtungsakt aus dem idioplasmatischen System ausgeschlossen, als unwirksam beseitigt 
und zu Ernährungsplasma umgewandelt werde“. Zugunsten dieser Annabme lässt sich anführen, dass, wie Van der Stricht und seine Schüler gezeigt haben, ein Teil der Mitochondrien der Eizelle tatsächlich zu Dotterkörnern umgewandelt wird.

Eihe weitere Möglichkeit, wie die wünschenswerte Gleichheit in bezug auf die Menge der männlichen und weiblichen Chondriosomen nachträglich erzielt werden könnte, besteht offenbar darin, dass die ersteren im Körper der Eizelle heranwachsen und sich vermehren.

Eine gleichwertige Verteilung der Erbmassen auf die aus der Eizelle hervorgehenden Zellen wird nach $\mathrm{O}$. Hertwig durch zahireiche Tatsachen der Zeugung und Regeneration unumgänglich verlangt.

Nun kann zwar im allgemeinen nicht davon die Rede sein, dass die Chondriosomen bei der Zellteilung eine "peinlich genaue Halbierung" nach der Art des Chromatins erfahren. Immerhin werden sie wohl stets wenigstens annähernd gleich auf die Tochterzellen verteilt (in den Spermatocyten einiger Tiere zeigen sie sogar "Andeutungen einer Sondermitose“). Jede bei der Teilung entstehende grössere Ungleichheit in der Menge kann aber durch vermehrtes oder vermindertes Wachstum leicht beseitigt werden.

Als ein sehr wichtiges Moment in der Beweisführung betrachtet $\mathrm{O}$. Hertwig schliesslich den dritten Punkt, nämlich die Verhütung der Summierung der Erbmassen. Naegeli hat schon gesagt: „Wenn bei jeder Fortpflanzung durch Befruchtung das Volumen des irgendwie beschaffenen Idioplasmas sich verdoppelte, so würden nach nicht sehr zahlreichen Generationen die Idioplasmakörper so sehr anwachsen, dass sie selbst einzeln nicht mehr in einem Spermatozoid Platz fänden." N a egeli bezeichnet es daher als notwendig, "dass bei der digenen Fortptlanzung die Vereinigung der elterlichen Idioplasmakörper erfolge, ohne eine den vereinigten Massen entsprechende dauernde Vergrösserung dieser materiellen Systeme zu verursachen“.

Bei der Kernsubstanz wird nun bekanntlich eine Summierung durch einen Reduktionsprozess verhütet, „durch welchen sie jedes Mal vor der Befruchtung auf die Hälfte verkleinert wird". Gibt es etwas entsprechendes auch bei den Chondriosomen? 
Im Hoden wird tatsächlich, wie D u esberg (1907) betont, eine Massenreduktion der Chondriosomen durch die beiden unmittelbar aufeinander folgenden Spermatocytenteilungen bewirkt. Bei den Richtungsteilungen der Eier dagegen eirscheint sie ausgeschlossen, weil die Mitochondrien im Zellleib verstreut bleiben und die beiden Teilungsprodukte eine sehr ungleiche Grösse haben.

Nun gibt es aber noch einen anderen Weg als denjenigen der vorherigen Halbierung; wie eine Summierung der Erbmassen verhütet werden könnte. Dieser Weg ist von Naegeli gezeigt worden. Na geli (S.220-230) lässt das männliche und weibliche Idioplasma sich bei der Befruchtung zu einem Mischprodukt vereinigen ${ }^{1}$ ). Der Forderung, dass dabei eine den vereinigten Massen entsprechende danernde Verdoppelung des idioplasmatischen Systems verhindert werde, kann nach $\mathrm{N}$ a egeli wohl nur durch die Annahme genügt werden, dass das Idioplasma aus Strängen besteht und dass der Querschnitt der durch Verschmelzung entstehenden kindlichen Idioplasmastränge in bezug auf die Zahl der Micellreihen im allgemeinen unverändert bleibt. Je ein väterlicher und mütterlicher Idioplasmastrang müssen sich demnach bei der Befruchtung zu einem solchen von doppelter Länge vereinigen; diese Vereinigung erscheint dann als der erste Schritt des individuellen Wachstums.

Was das Zustandekommen der Verschmelzung anlangt, so stellt Naegeli sich vor, dass männliche und weibliche Idioplasmakörper sich gegenseitig in derselben Weise wie Eizelle und Spermium anziehen und sich infolge davon aneinanderlegen. Weiter lösen sich entweder Micelle von dem einen System nach und nach $a b$ und wandern in das andere hinüber; oder aber männliche und weibliche Idioplasmakörper bleiben intakt und nwirken bloss gegenseitig auf das Wachstum der einen und anderen so ein, dass dasselbe zu einer mittleren Bildung hinstrebt". Mit

1) Man vergleiche auch, was Hensen 1895 (S. 748-749) geschrieben hat: „Nach den bisher vorliegenden Thatsachen kommt man zu dem Schluss, dass durch die Summierung von väterlichem und mütterlichen Erbteil der Erbstücke im Ei z u viele werden und daher die Verminderung derselben stattzufinden hat. In der Wissenschaft würde man kanm Bedenken gehabt haben, bei diesem Anlass an eine Verschmelzung gleichwertiger Erbformen zu denken, wenn nicht die Tatsache einer Ausstossung von Kernmasse vor und während der Befruchtung den Gedanken so überaus nahe gelegt hätte, dass hier eine Halbierung stattfinde." 
Bezug auf die Details dieser von $\mathrm{Naegeli}$ in bewunderungswürdiger Weise bis ins kleinste ausgearbeiteten Vorgange muss ich auf das Original verweisen.

Ich resumiere zum Schluss dahin, dass die Chondriosomen Erbmasse darstellen könnten, trotzdem sie den Bedingungen, welche die Kernsubstanz in ihrer Eigenschaft als solche erfüllt, nicht oder jedenfalls nicht von vornherein oder in anderer Weise genügen.

\section{IX. Über zwei neuere Versuche, das Vererbungs- monopol des Kerns zu erweisen.}

Neuerdings sind von Boveri und von Strasburger Beobachtungen beschrieben worden, welche beweisen sollen, dass lediglich der Kern der Vererbungsträger sei.

Boveri (1904, S. $106-110$ und 1907, S. 111 u. folg.) schliesst dies ans den Eigenschaften von Seeigellarven, welche aus "dreiteiligen" Eiern hervorgegangen sind.

Doppeltbefruchtete Seeigeleier teilen sich zwar typischer Weise simultan in vier Zellen; es lässt sich aber durch Eingriffe, auf die hier nicht näher eingegangen werden soll, erzielen, dass nur drei Pole entstehen und demgemäss eine simultane Dreiteilung eintritt. Falls, was durchaus möglich ist, zwischen je zwei der drei Pole einer der drei Vorkerne gelangen würde, wären die Chromatinverhältnisse bei dieser Art dispermer Entwicklung insofern nahezu normal, als ,jede Zelle die Normalzahl von Chromosomen und jede Chromosomenart doppelt besitzen" würde, wie aus nebenstehendem Schema ersichtlich ist:

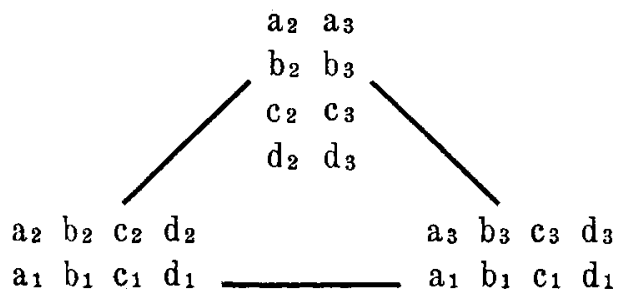

Jedoch erhält das eine Drittel des Keimes Chromatin des Eikerns und des einen Spermakerns, das zweite solche des Eikerns und des anderen Spermakerns, das dritte Chromatin der beiden Spermakerne. "Sind sonach die Chromosomen für die spezifische Gestaltung der Larvencharaktere massgebend, so ist $\mathrm{zu}$ erwarten, 
dass die Plutei aus doppeltbefruchteten Eiern in ihren einzelnen Bereichen einen verschiedenen individuellen Typus darbieten. In der Tat ist es ein fast durchgehendes Charakteristikum der aus simultan dreiteiligen Eiern hervorgegangenen Plutei, dass sie. mehr oder weniger asymmetrisch sind."

Gegen den Schluss, "dass die Verschiedenheit in der Ausbildung der einzelnen Keimbereiche durch die Verschiedenheit der Kernsubstanz bedingt sei", lässt sich nun aber ein Einwand erheben, welchen Boveri selbst folgendermaßen formuliert: "dass in einem Bereich der Larve Protoplasma des einen, in einem anderen Protoplasma des anderen Spermiums anwesend sei, und dass dieses verschiedene Spermaprotoplasma an der verschiedenartigen Entwicklung einzelner Bereiche schuld sei". Boveri erklärt es jedoch für ausgeschlossen, dass das Spermaprotoplasma, von dem übrigens bei Echiniden nichts zu sehen sei, eine derartige Wirkung haben könne. „Denn es müsste dann auch bei der normalen monospermen Befruchtung diese Rolle spielen, und da es - dies vorausgesetzt - diese bestimmende Wirkung hier in allen Bereichen des neuen Organismus ganz gleichmässig ausübt, so müssten Mittel vorhanden sein, durch die es, gleich dem Spermachromatin, in identischer Weise auf alle Tochterzellen verteilt wird. Solche Mittel bestehen, wie uns die Falle lehren, wo das Spermaprotoplasma wahrnehmbar ist (Ascaris), nieht. Damit dürfte es ausgeschlossen sein, ihm überhaupt eine so bedeutungsvolle, aufs feinste arbeitende Wirkung zuzuschreiben. Wollte man aber annehmen, dass sich die Vererbungstendenzen des Spermaprotoplasmas sofort dem ganzen Ei gleichmässig mitteilen, so müssten natürlich bei Anwesenheit zweier Spermien deren beiderseitige Qualitäten gleichfalls ganz gleichmässig gemischt auf das Ei übergehen, so dass gerade bei dieser Annahme die charakteristische Asymmetrie der dispermen Larven völlig unerklärt bliebe‘:

Diese Argumentation Boveris vermag ich nicht als stichhaltig anzuerkennen. Wenn wir die Vorstellungen, welche $\mathrm{Naegeli}$ sich von der Beschaffenheit seines Idioplasmas gebildet hat, auf die Chondriosomen übertragen, welche nach meiner Annahme die Trăger der cytoplasmatischen Vererbung sind, so ist es übertlüssig, zu verlangen, dass sie nach Art des Chromatins "in identischer Weise" auf die Tochterzellen verteilt werden sollen. Es ist mehr als genug, wenn sie in zwei annahernd 
gleiche Hälften zerlegt werden. Welche „Mittel “ aber speziell im Ascarisei für die Verteilung der Chondriosomen bestehen, darüber sind wir einstweilen, auf Grund der Methoden, die sich bisher bei diesem Objekt haben anwenden lassen, nicht imstande irgend etwas auszusagen. Jedenfalls dürften diese Mittel dafür genügen, „dass sich die Vererbungstendenzen des Spermaprotoplasmas sofort dem ganzen Ei gleichmässig mitteilen ". Andererseits aber ist es sehr wohl möglich, dass in einem doppelt befruchteten Seeigelei, welches eine Dreiteilung erfährt, die sämtlichen Chondriosomen eines der beiden Samenfäden auf einen Bezirk des Eies beschränkt bleiben; die "charakteristische Asymmetrie der dispermen Larven" könnte einzig und allein auf diesen Umstand zurückzuführen sein.

St rasburger hat das Verhalten der Spermakerne in den Pollenschläuchen von Angiospermen, welches ihn 1884 zu der Erklärung bestimmte, dass das Cytoplasma an der Befruchtung nicht beteiligt sei, 1908 von neuem geprüft. Er vermochte auch bei Anwendung moderner Technik und Untersuchung mit Apochromaten um die Spermakerne in den Pollenschlauchenden von Lilium kein "Eigenplasma" abzugrenzen; für den Befruchtungsvorgang stände somit nur der gemeinsame cytoplasmatische Inhalt des Pollenschlauches zur Verfügung, falls doch noch Cytoplasma zur Übertragung erblicher Eigenschaften des Vaters auf die Nachkommen notwendig sein sollte. Ein Erguss von cytoplasmatischem Pollenschlauchinhalt in das $\mathrm{Ei}$ ist aber in keinem Fall bisher beobachtet worden. In dieses schlüpft vielmehr nur ein nackter Spermakern ein, um sich mit dem Eikern zu vereinigen. Die Sichtung der Erscheinungen, welche bei Angiospermen die Befruchtung vorbereiten, erlaubt daher, nach Strasburgers Ansicht, nur den einen Schluss, dass die Kerne die Träger der erblichen Eigenschaften sind.

Da nun aber das Cytoplasma des Pollenschlauchs den Spermakern, wie Strasburger (1908, S. 540) selbst sagt, "an seinen Bestimmungsort befördert", so lässt es sich meines Erachtens nicht ausschliessen, dass etwas davon mit in das Ei hineingelangt. Ferner ist aber die Möglichkeit nicht abzuweisen, dass schon ein einziges winziges Mitochondrium genügen könnte, um die Eigenschaften des väterlichen Cytoplasmas auf dasjenige des Eies zu übertragen. 


\section{Literaturverzeichnis.}

Altmann, R., 1890: Die Elementarorganismen und ihre Beziehungen zu den Zellen. Erste Aufl. (1894 zweite Aufl.).

Arnold, J., 1900, 1: Über Fettkörnchenzellen, ein weiterer Beitrag zur Granulalehre. Virchows Arch., Bd. 161.

Derselbe, 1900, 2 : Über Siderosis und siderofere Zellen, zugleich ein Beitrag zur Granulalehre. Ebenda.

Derselbe, 1902: Über Phagocytose, Synthese und andere intrazellulüre Vorgänge. Münchn. med. Wochenschr.

Derselbe, 1905, 1: Die Morphologie der Milch- und Kolostrumsekretion, sowie deren Beziehung zur Fettsynthese, Fettphagocytose, Fettsekretion und Fettdegeneration. Beiträge z. pathol. Anat.. Bd. 38.

Derselbe, 1905, 2: Über Bau und Sekretion der Drüsen der Froschhaut, zugleich ein Beitrag zur Plasmosomen-Granulalehre. Arch. f. mikr. Anat. u. Entwicklungsgesch., Bd. 65.

Derselbe, 1907, 1: Die Rolle der Zellgranula bei der hämatogenen Pigmentierung und Bemerkungen über entzündliche Zellformen. Virchows Arch., Bd. 196.

Derselbe, 1907, 2: Plasmosomen, Granula, Mitochondrien, Chondriomiten und Netzfiguren. Anat. Anz., Bd. 31.

Ballowitz, E, 1900: Über das Epithel der Membrana elastica posterior des Auges, seine Kerne und eine merkwürdige Struktur seiner grossen Zellsphären. Arch f. mikr. Anat. u. Entwicklungsgesch., Bd. 56 .

Van Beneden, E., 1883: Recherches sur la maturation de l'oeuf, la fécondation et la division cellulaire.

Derselbe und Neyt, A., 1887: Nouveiles recherches sur la fécondation et la division mitosique chez l'Ascaride mégalocéphale. Bull. de l'acad. de Belgique, sér. 3 , t. 14.

Benda, C., 1897: Neuere Mitteilungen über die Histogenese der Säugetierspermatozoen. Verh. d. phys. Ges. zu Berlin, Jahrg. 1896/1897.

Derselbe, 1898, 1: Über die Entstehung der Spiralfaser des Verbindungsstückes der Sängetierspermien. Verh. d. anat. Ges. in Kiel.

Derselbe, 1898, 2: Über die Spermatogenese der Vertebraten und höherer Evertebraten. Verh. d. phys. Ges. zu Berlin, Jahrg. 1897/1898.

Derselbe, 1899, 1: Weitere Mitteilongen über die Mitochondria. Verh. d. phys. Ges. zu Berlin, Jahrg. 1898/1899.

Derselbe, 1899, 2: Weitere Beobachtungen über die Mitochondria und ihr Verhältnis zu Sekretgranulationen nebst kritischen Bemerkungen. Verh. d. phys. Ges. zu Berlin, Jahrg. 1899/1900.

Derselbe, 1903: Die Mitochondria. Erg. d. Anat. u. Entwicklungsgesch., Bd. 12, 1902.

v. Bergen, Fr., 1904 : Zur Kenntnis gewisser Strukturbilder („Netzapparate", "Saftkanälchen ", "Trophospongien ") in Protoplasma verschiedener Zellenarten. Arch. f. mikx. Anat. u. Entwicklungsgesch., Bd. 64. 
Bergh, R. S., 1892 (dat. Nov. 1891): Kritik einer modernen Hypothese von der Übertragung erblicher Eigenschaften. Zool. Anz.

Berthold, G., 1886: Studien über Protoplasmamechanik

Besta, 1904, 1: Ricerche intorno alla genesi e al modo di formazione della cellula nervosa. Rivista sperimentale di Freniatria, vol. 30.

Derselbe, 1904, 2: Ricerche intorno al modo con cui si stabilino i rapporti mutui tra gli elementi nervosi embrionali. Ebenda.

Boveri, Th, 1887: Über den Anteil des Spermatozoons an der Teilung des Eies. Sitzungsber. d. Ges. f. Morph. u. Phys. zu München, Bd. 3.

Derselbe, 1889: Ein geschlechtlich erzeugter Organismus ohne mütterlichen Anteil. Sitzungsber. d. Ges. f. Morph u Phys., Bd. 5.

Derselbe, 1892 : Befruchtung. Erg. d. Anat. u. Entwicklungsgesch., Bd. 1, 1891.

Derselbe, 189ã: Über das Verhalten der Centrosomen bei der Befruchtung des Seeigeleies, nebst allgemeinen Bemerkungen über Centrosomen und Verwandtes. Verhandl. d. physilr.-med. Ges. zu Würzburg

Derselbe, 1903: Über den Einfluss der Samenzelle auf die Larvencharaktere der Echiniden. Arch. f. Entwicklungsmech., Bd. 16.

Derselbe, 1904: Ergebnisse über die Konstitution der chromatischen Substanz des Zellkerns.

Derselbe, 1907: Zellen-Studien, Heft 6. Die Entwicklung dispermer Seeigeleier. Ein Beitrag zur Befruchtungslehre und zur Theorie des Kerns.

v. Brunn, A., 1884: Beiträge zur Kenntnis der Samenkörper und ihrer Entwicklung bei den Säugetieren und Vögeln. Arch. f. mikr. Anat., Bd. 23.

Bütschli, O., 1871, 1: Vorläufige Mitteilung über Bau und Entwicklung der Samenfäden bei Insekten und Crustaceen. Zeitschr. f. wiss. Zool., Bd. 21 .

Derselbe, 1871, 2: Nähere Mitteilungen über die Entwicklung und den Bau der Samenfäden der Insekten. Zeitschr. f. wiss. Zool., Bd. 21.

Cajal, S. R, 1908: Nouvelles observations sur l'évolution des neuroblastes, avec quelques remarques sur l'hypothèse neurogénètique de HensenHeld. Anat. Anz, Bd. 32

Conklin, E. G., 1905, 1: The Organization and Cell-Lineage of the Ascidian Eggs. Journ. of the Acad. of Nat. Sc. of Philadelphia, vol. 13.

Derselbe, 1905, 2: Organ-forming Substances in the Eggs of Ascidians. Biol. Bull., vol. 8 .

Derselbe, 1905, 3: Mosaic Development in Ascidian Eggs. Journ. of Experimt. Zool, vol. 2.

Crampton, H. E., 1896: Experimental Studies on Gasteropod Development. Arch. f. Entwicklungsmech., Bd. 3.

Czermak, J. N., 1879: Über die-Spermatozoiden von Salamandra atra. Ein Beitrag zur Kenntnis der festen F'ormbestandteile im Samen der Molche. Gesammelte Abhandlungen, Bd. 1.

Driesch, H., 1897: Betrachtungen über die Organisation des Eies und ihre Genese. Anhang III. Einiges über die Organisation des Eies und über die ersten Entwicklungsvorgänge von Myzostoma. Arch. f. Entwicklungsmech, Bd. 4 . 
Derselbe, 1898:. Über rein mütterliche Charaktere an Bastardlarven von Echiniden. Arch. f. Entwicklungsmech., Bd. 7.

Derselbe, 1903: Über Seeigelbastarde. Arch. f. Entwicklungsmech., Bd. 16.

Derselbe, 1905̃: Die Entwicklungsphysiologie von 1902 bis 1905. Erg. d. Anat. u. Entwicklungsgesch., Bd. 14, 1904.

Derselbe und Mnrgan, P. H., 1896: Zur Analysis der ersten Entwicklungsstadien des Ctenophoreneies. II. Von der Entwicklung ungefurchter Eier mit Protoplasmadefekten. Arch. f. Entwicklungsmech., Bd. 2.

Duesberg, J., 1907: Der Vitochondrial-Apparat in den Zellen der Wirbeltiere und Wirvellosen. Arch. f. mikr. Anat. u. Entwicklungsgesch, Bd. 71

Van Durme, XI., 1907: Les mitochondries et la méthode de Sjüvall dans l'ovogenèse des oiseaux. Ann. de la Suc. de Méd. de Gand, vol. 87.

Fick, R., 1892: İber die Befruchtung des Axolotleies. Anat. Anz., Jahrg. 7.

Derselbe, 1893: ̈̈ber die Reifung und Befruchtung des Axolotleies. Zeitschr. f. wiss. Zool., Bd. 56 .

Fischel, A., 1\%02: Über den gegenwürtigen Stand der experimentellen Teratologie. Verh. d. Deutsch. path. Ges., V. Tagung zu Karlsbad.

Derselbe, 1903: Entwick!ung nnd Organdifferenzierung. Arch. f. Entwicklungsmech., Bd. 1 j.

Flemming, W, 1882. 1: Beiträge zur Kenntnis der Zelle und ihrer Lebenserscheinungen. III. Teil. Arch. f. mikr. Anat., Bd. 20 .

Derselbe, 1882, 2: Zellsubstanz, Kern und Zellteilung.

Derselbe, 1884: Ïber Bauverhältnisse, Befruchtung und erste Teilung der tierischen Eizelle. Biol. Zentralbl., Bd. 3.

Derstlbe, 1891: Zur Entwicklungsgeschichte der Bindegewebsfibrillen. Internat. Beitr. zur wiss. Med., Festschr. f. R. Virchow.

Derselbe, 1897: Über den Bau der Bindegewebszellen und Bemerkungen über die Struktur der Zellsubstanz im allgemeinen. Zeitschr. f. Biologie, Bd. 34, N. F. 16

Derselbe, 1899: Eröffnungsrede. Verh. d. anat. Ges. in Tübingen.

Fol, H., 1891 : Die Centrenquadrille, eine neue Episode aus der Befruchtungsgeschichte. Anat. Anz., Jahrg. 6.

Frenzel, J., 1886: Das Idioplasma und die Kernsubstanz. Ein kritischer Beitrig zur Frage nach dem Vererbungsstoff. Arch f. mikr. Anat, Bd. 27 .

Goldschmidt, R., 1904: Der Chromidialapparat lebhaft funktionierender Gewebszellen. (Histologische Untersuchungen an Nematoden II.) Zool. Jahrb., Abt. f. Anat. u. Ontog., Bd. 21.

Golgi, C., 1898: Sur la structure des cellules nerveuses. Arch. ital. de Biul., t. 30.

Godlewsky, E., 1906 : Untersuchungen über die Bastardierung der Echinidenund Crinoidenfamilie. Arch. f. Entwicklungsmech., Bd. 20.

Held, H.. 1905: Die Entstehung der Neurofibrillen. Yeurol. Zentralbl., Jahrg. 24. 
Henking, H., 1891: Cntersuchungen über die ersten Entwicklungsvorgänge in den Eiern der Insekten. II. Über Spermatogenese nnd deren Beziehung zur Eientwicklung bei Pyrrhocoris apterus L. Zeitschr. f. wiss. Zool., Bd. ̌́1.

Hensen, V., 1881: Physiologie der Zeugang. Handbuch der Physiologie, Bd. 6, II. Teil.

Derselbe, 1885: Die Grundlagen der Vererbung nach dem gegenwärtigen Wissenskreis. Landwirtschaftl. Jahrbiicher, Bd. 14.

Hertwig, 0., 1875: Beiträge zur Kenntnis der Bildung, Befruchtung und Teilung des tierischen Eies. I. Abt. Morpholog. Jahrb., Bd. 1.

Derselbe, 1885 (dat. Okt. 1884): Das Problem der Befruchtung and der Isotropie des Eies, eine Theorie der Vererbung. Jenaische Zeitschr. f. Naturw., Bd. 18 (N. F., Bd. 11).

Derselbe, 1893: Die Zelle und die Gewebe.

Derselbe, 1906: Allgemeine Biologie. Zweite Auflage des Lehrbuchs „Die Zelle und die Gewebe*.

D'Hollander, F., 1904: Les pseadochromosomes dans les oogonies et les oocytes des Oiseaux. Bibl. anat., t. 13.

v. Koelliker, A., 1885: Die Bedeutung der Zellkerne für di. Vorgänge der Vererbung. Zeitschr. f. wiss. Zool, Bd. 42.

Kopsch, Fr., 1902: Die Darstellung des Binnennetzes in spinaien Ganglienzellen und anderen Körperzellen mittels Osmiumsäure. Sitzangsber. d. K. Preuss. Akad. d. Wiss. zu Berlin, Bd. 40.

Lams, H., 1904: Contribution à l'étude de la genèse da vitellus dans l'ovule des Teléostéens. Arch. d'anat. micr., t. 6.

Derselbe, 1907: Contribution à l'étude de la genèse du vitellus dans l'ovule des Amphibiens (Rana temporaria). Ebenda, t. 9.

v. Lenhossêk, M., 1903: Die Entwicklung des Glaskörpers.

Loeb, J., 1903: Über die Befruchtung von Seeigeleiern durch Seestermsamen. II. Mitteilung. Arch. f. d. ges. Phys., Bd. 99.

Derselbe, 1906: Vorlesungen über die Dynamik der Lebenserscheinungen.

M ehnert, E., 1898: Biomechanik, erschlossen aus dem Prinzipe der Organogenese.

Metzner, Rud., 1890: Über die Beziehungen der Granula zum Fettansatz. Arch. f. Anat. u. Phys., Anat. Abt.

Meves, Fr., 1897: Über Struktur nnd Histogenese der Samenfäden von Salamandra maculosa. Arch. f. mikr. Anat., Bd. 亏0.

Derselbe, 1898: Über das Verhalten der Zentralkörper bei der Histogenese der Samenfäden von Mensch and Ratte. Verh. d. anat. Ges. in Kiel.

Derselbe, 1899: Über Struktur und Histogenese der Samenfäden des Meerschweinchens. Arch. f. mikr. Anat., Bd. 54.

Derselbe, 1900: Über den von v. la Valette St. George entdeckten Nebenkern (Mitochondrienkörper) der Samenzellen. Arch. f. mikr. Anat. u. Entwicklungsgesch., Bd. 56 .

Derselbe, 1904: Über das Vorkommen von Mitochondrien bezw. Chondromiten in Pflanzenzellen. Ber. d. Deutsch. bot. Ges., Bd. 22. 
Derselbe, 1907, 1: Die Spermatocytenteilungen bei der Honigbiene (Apis mellifica L.), nebst Bemerkungen über Chromatinreduktion. Arch. f. mikr. Anat. v. Entwicklungsgesch., Bd. 70.

Derselbe, 1907, 2: Über Mitochondrien bezw. Chondriokonten in den Zellen junger Embryonen. Anat. Anz., Bd. 31.

Derselbe, 1907, 3: Die Chondriokonten in ihrem Verbältnis zur Filarmasse Flemmings. Anat. Anz., Bd. 31.

Derselbe und Duesberg, J., 1907: Die Spermatocytenteilungen bei der Hornisse (Vespa crabro L.). Arch. f. mikr. Anat. u. Entwicklungsgesch., Bd. 71.

Mikosch, C., 1894: Über Strukturen im pflanzlichen Protoplasma. Verh. d. Ges. Deutsch. Naturf. a. Ärzte. 66. Versamml, zu Wien.

v. Nägeli, C., 1884 : Mechanisch-physiologische Theorie der Abstammungslehre.

Negri, A., 1900: Über die feinere Struktur der Zellen mancher Drüsen bei den Säugetieren. (Mitgeteilt von Golgi.) Verh. d. anat. Ges., Pavia.

Næmec, B., 1901: Die Reizleitung und die reizleitenden Strukturen bei den Pflanzen.

Nussbaum, M., 1883: Ascaris und Leptodera. Sitzungsber. d. Niederrh. Ges. f. Natur- u. Heilkunde in Bonn.

Derselbe, 1884: Über die Veränd̉erungen der Geschlechtsprodukte bis zur Eifurchnng; ein Beitrag zur Lehre der Vererbung. Arch. f. mikr. Anat., Bd. 23 .

Derselbe, 1886: ̈̈ber die Teilbarkeit der Materie. I. Mitteilung. Die spontanc and künstliche Teilung der Infusorien. Arch. f. mikr. Anat., Bd. 26.

Pensa, A., 1901: Osservazioni sulla struttura delle cellule cartilaginee. Rendic. d. R. Ist. Lomb. di Sc. e Lett., Ser. 2, vol. 34.

Pfeffer, W., 1886: Über Aufnabme von Anilinfarben in lebende Zellen. Untersuchungen aus dem botanischen Institut zu Tübingen, Bd. 2.

Popoff, M., 1906: Zur Frage der Homologisierung des Binnennetzes der Ganglienzellen mit den Chromidien (= Mitochondria etc.) der Geschlechtszellen. Anat. Anz., Bd. 29.

Rabl, C., 1906: Über ,organbildende Substanzen“ und ihre Bedeutung fur die Vererbung.

Rauber, A., 1887: Personalteil und Germinalteil des Individuums. Zool. Anz.

Russo, A., 1907, 1: Sull' origine dei mitocondrî e sulla formazione del deutoplasma nell oocite di alcuni mammiferi. Rend. della R. Accad. dei Lincei, rol. 16.

Derselbe, 1907, 2: Modificazioni sperimentali dell' elemento epiteliale dell' ovaia dei mammiferi. R. Accad. dei Lincei, ser. 5 a, vol. 6.

Derselbe, 1908: Sulla origine e sulla funzione dell' apparato mitocondriale nelle cellule sessuali dei mammiferi. Boll. dell' Accad. Gioenia di Sc. Nat. in Catania.

Sachs, Jul., 1893: Stoff und Form der Pflanzenorgane. Gesammelte Abhandlungen über Pflanzenphysiologie. Zusatz 1892 auf S. 1202. 
v. Smirnow, A. E., 1906: Über die Mitochondrien und den Golgischen Bildungen analoge Strakturen in einigen Zellen von Hyacintbus orientalis. Anat. Hefte, I. Abt., Bd. 32.

De Somer, Eug., 190\%: Les premiers stades de la vitellogenèse dans l'orule de la Poule. Ann. de la Soc. de méd. de Gand, vol. 85.

Strasburger, E., 1877: ̈̈ber Befruchtung und Zellteilung. Jenaische Zeitschr. f. Naturw., Bd. 11 (N. F., Bd. 4).

Derselbe, 1.884, 1: Neue Untersuchungen über den Befruchtungsvorgang bei den Phanerogamen als Grundlage für eine Theorie der Zeugung.

Derselbe, 1884, 2: Das botanische Praktikum.

Derselbe, 1907, 1: Ontogenie der Zelle seit 1875. Progressus rei botanicae, Bd. 1.

Derselbe, 1907, 2: Über die Individualität der Chromosomen und die Propfhybriden-Frage. Jahrb. f. wiss. Bot., Bd. 44.

Derselbe, 1908: Chromosomenzahlen, Plasmastrukturen, Vererbungstrïger und Reduktionsteilung. Jahrb. f. wiss. Bot., Bd. $4 \dot{3}$.

Van der Stricht, 0., 1902: Les "Pseudochromosomes" dans l'oncyte de Chauve-souris. C. R. de l'Assoc. des anatomistes, Montpellier.

Derselbe, 1904, 1: La couche vitellogène et les mitochondries de l'oeuf des Yammifères. Verh. d. anat. Ges., Jena.

Derselbe, 190ł, 2: La structure de l'oeuf des Mammiferes. Première partic: L'oocyte au stade de l'accroissement. Arch. de biologie, t. 21 .

Derselbe, 190̄̃, 1: La structure de l'oeuf des Mammiferes. Seconde partie: Structure de l'oeuf ovarique de la femme. Bull. de l'Acad. R. de méd. de Belgique.

Derselbe, 1905, 2: La structure de l'oeuf de Chauve-souris (V. noctula). Verh. d. anat. Ges., Genf.

Tischler, G., 1906: Über die Entwicklung des Pollens und der Tapetenzellen bei Ribes-Hybriden. Jabrb. f. wiss. Bot., Bd. 42.

Toyama, K., 1894: On the Spermatogenesis of the Silk-Worm. Bull. of the Agricultural Coll., Imp. Univ., Tokyo, Japan.

v. la Valette St. George, 1867: Über die Genese der Samenkörper. Arch. f. mikr. Anat., Bd. 3.

Derselbe, 1886: Spermatologische Beiträge. Zweite Mitteilung. Arch. f. mikr. Anat., Bd. 27.

Verworn, M., 1891: Die physiologische Bedeutung des Zellkerns. Arch. f. d. ges. Physiologie, Bd. 51, 1892.

Waldeyer, W., 1888: C̈ber Karyokinese und ihre Beziehungen zu den Befruchtungsvorgängen. Arch. f. mikr. Anat., Bd. 32.

Derselbe, 1893: Eröffnungsrede. Verh. d. anat. Ges. in Göttingen.

Derselbe, 1895: Die neueren Ansichten über den Bau und das Wesen der Zelle. Deutsche med. Wochenschrift, No. 43.

Weigert, C., 1887: Neuere Vererbungstheorien. Schmidts Jahrb. d. gesamten Medizin, Bd. 21 o.

Weismann, A., 1885: Die Kontinuität des Keimplasmas als Grundlage einer Theorie der Vererbang. 
Wilson, E. B., 1904: Experimental Studies on germinal Localization. I. The Germ-regions in the Egg of Dentalium. II. Experiments on the Cleavage-mosaic in Patella and Detalium. Journ. of Experiment. Zoology, vol. 1.

Derselbe und Mathews, 1895: Maturation, Fertilisation and Polarity in the Echinoderm Egg. Journ. of Morph., vol. 5.

Zacharias, E., 1888: Über Kern- und Zellteilung. Botan. Zeitg.

\section{Erklärung der Abbildungen auf Taf. XXXIX-XLII.}

Die Figuren der Taf. XXXIX sind mit Zeiss' Apochromat $2 \mathrm{~mm}$ (Apertur 1,30 oder 1,40) und Komp.-Ok. 18, diejenigen der Tafeln XL-XLII mit demselben Objektiv und Komp.-Ok. 6 unter Benutzung des A b beschen Zeichenapparates entworfen. Der Abstand der Zeichenebene von der Tischplatte betrug $171 / 2 \mathrm{~cm}$. Sämtliche Figuren stammen von Hühnerenbryonen, die in $\mathrm{Fle} \mathrm{mm}$ in $\mathrm{g}$ schem Gemisch von der oben angegebenen Zusammensetzung fixiert und mit Eisenhämatoxylin nach $\mathrm{M}$. $\mathrm{H}$ e id e $\mathrm{nh}$ a in gefärbt sind.

\section{Tafel XXXIX.}

Fig. 1. Von einem Hühnerembryo von ca. 20 Stunden. Stück eines Querschnitts durch die Primitivrinne, seitlich von derselben. ak, $\mathrm{mk}$, ik: äusseres, mittleres, inneres Keimblatt.

Fig. 2-17. Von einem Hühnerembryo von ca. 27 Stunden (mit ca. 8 Urwirbeln).

Fig. 2. Gruppe von 3 Epidermiszellen.

Fig. 3-6. Epidermiszellen in Mitose. Fig. 3 Mutterstern, Fig. 4 Doppelstern mit verklumpten Chromosomen, Fig. 5 und 6 Zellleib durchgeschnürt.

Fig. 7. Gruppe von Zellen aus der Wand des Medullarrohrs.

Fig. 8. Obere Zellschicht: Ektoderm (ak), untere: Hautfaserblatt (df; sollte heissen hf); ans dem Bereich der area opaca.

Fig. 9-12. Mesenchymzellen, Fig. 9 und 10: im Ruhezustand, Fig. 11 und 12: in Mitose.

Fig. 13. Zellen der Herzwandung, durch Querbrücken zusammenbüngend.

Fig. 14. Fünf benachbarte Zellen aus einer "Blutinsel".

Fig. 15-16. Junge, soeben frei gewordene Blutzellen aus dem Innern einer Gefässanlage.

Fig. 17. Entodermzelle aus der Peripherie der area pellucida.

Fig. 18-26. Von einem Hühnerembryo von 2 Tagen und 1ó Stunden.

Fig. 18-23. Rote Blutzellen, Fig. 18-20 in Flächenansicht, Fig. 21 in Kantenansicht, Fig. 22 and 23 in Mitose.

Fig. 24. Gruppe von Darmepithelzellen.

Fig. 25. Schnitt durch ein Stück Hautmuskelplatte (c: Coriumblatt, $m$ : Muskelblatt) mit darüber gelagerter Epidermis (e).

Fig. 26. Gruppe von VIesenchymzellen aus dem Urwirbelkern. 
Tafel XI.

Fig. 27-29. Von demselben Embryo von ca. 27 Stunden, von dem auch Fig. 2-17 stammen.

Fig. 27. Querschnitt durch den hinteren Teil des Kopfes, hinter dem Eingang in die Kopfdarmhöhle.

Fig. 28. Schnitt durch die area pellucida und den inneren Teil der area opaca (Teil eines Querschnitts, der durch das hintere Ende der Primitivrinne hindurch gelegt ist). Das rechte Ende der Fig. 28 a setzt sich mit dem linken der Fig. $28 \mathrm{~b}$ fort. ak, mk, ik: äusseres, mittleres, inneres Keimblatt.

\section{Tafel XII.}

Fig. 30. Von demselben Embryo von ca. 27 Stunden, von dem auch Fig. 2-17 und Fig. 27-29 stammen. Querschnitt durch den vorderen Teil der Herzanlage. m Medullarrobr, ch Chorda, d Darmhöhle, h Herzendothel, me myoepicardialer Mantel, df Darmfaserblatt, ik inneres Keimblatt.

Fig. 31. Von demselben Embryo von 2 Tagen und 15 Stunden, von dem auch die Fig. 18-26 stammen: Schnitt durch die Rückengegend.

\section{Tafel XLII.}

Fig. 32. Von einem Embryo von ca. 50 Stunden. Teil eines Schnittes durch die Augenanlage. c Cornea, vl vordere, hl hintere Wand der Linsenblase; aa äussere, ia innere Lamelle der sekundären Augenblase.

Fig, 33. Von demselben Embryo von 2 Tagen und 15 Stunden, von dem auch die Fig. 18-26 und Fig. 31 stammen: Schnitt durch die Darmwand; de Darmepithel, df Darmfaserblatt.

Fig. 34-37. Von einem Embryo von 3 Tagen und 9 Stunden.

Fig. 34. Schnitt durch die Wandung des Herzohrs.

Fig. 35. Querschnitt durch den dorsalen Teil einer Rückenmarkshälfte.

Fig. 36. Schnitt durch ein Spinalganglion (g) mit umgebendem Mesenchymgewebe (m) und ein Stück Rückenmark (r) mit der austretenden vorderen Nervenwurzel (vw); bl Blutgefässe.

Fig. 37. Hälfte eines Querschnitts durch die Chorda. 


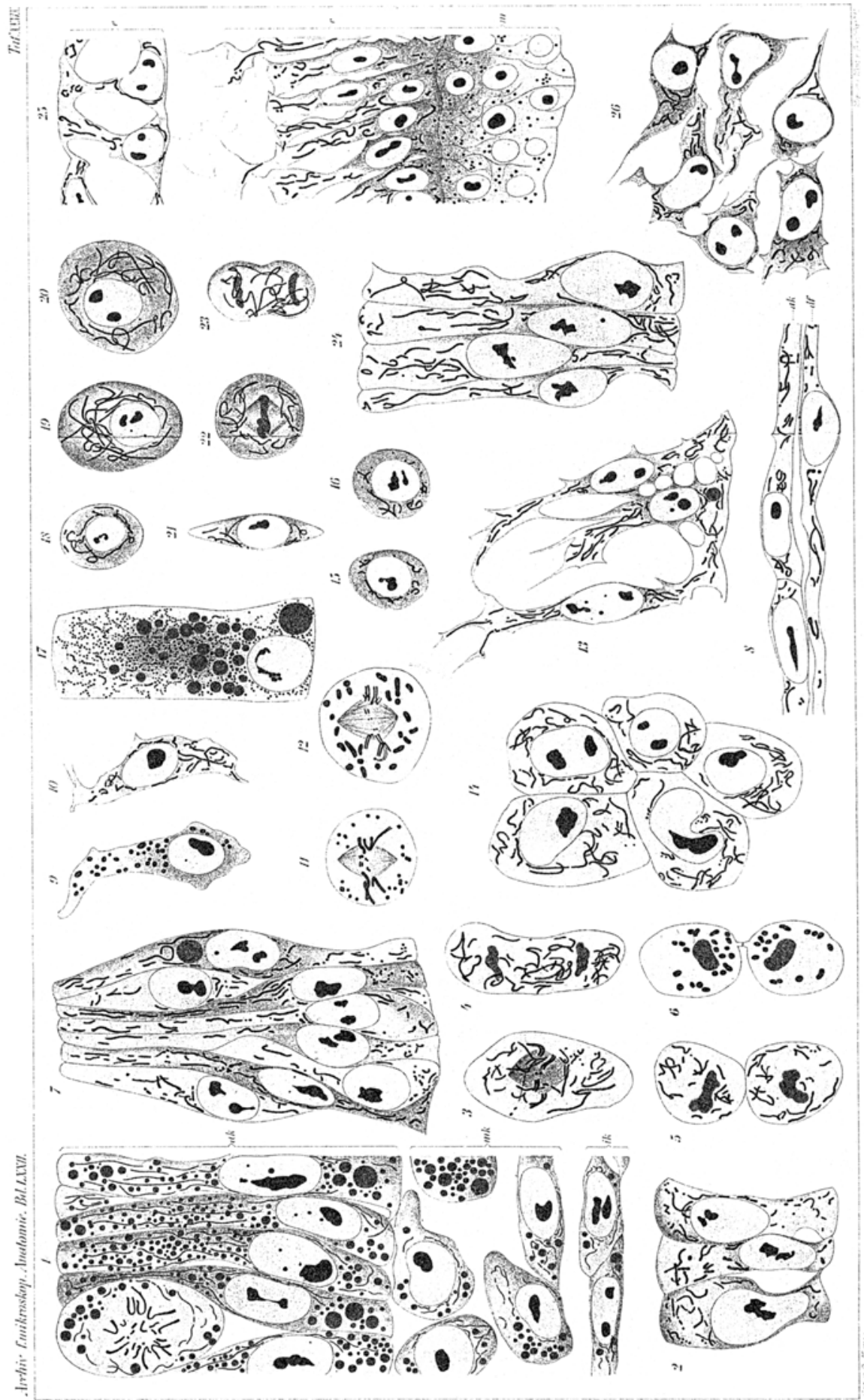




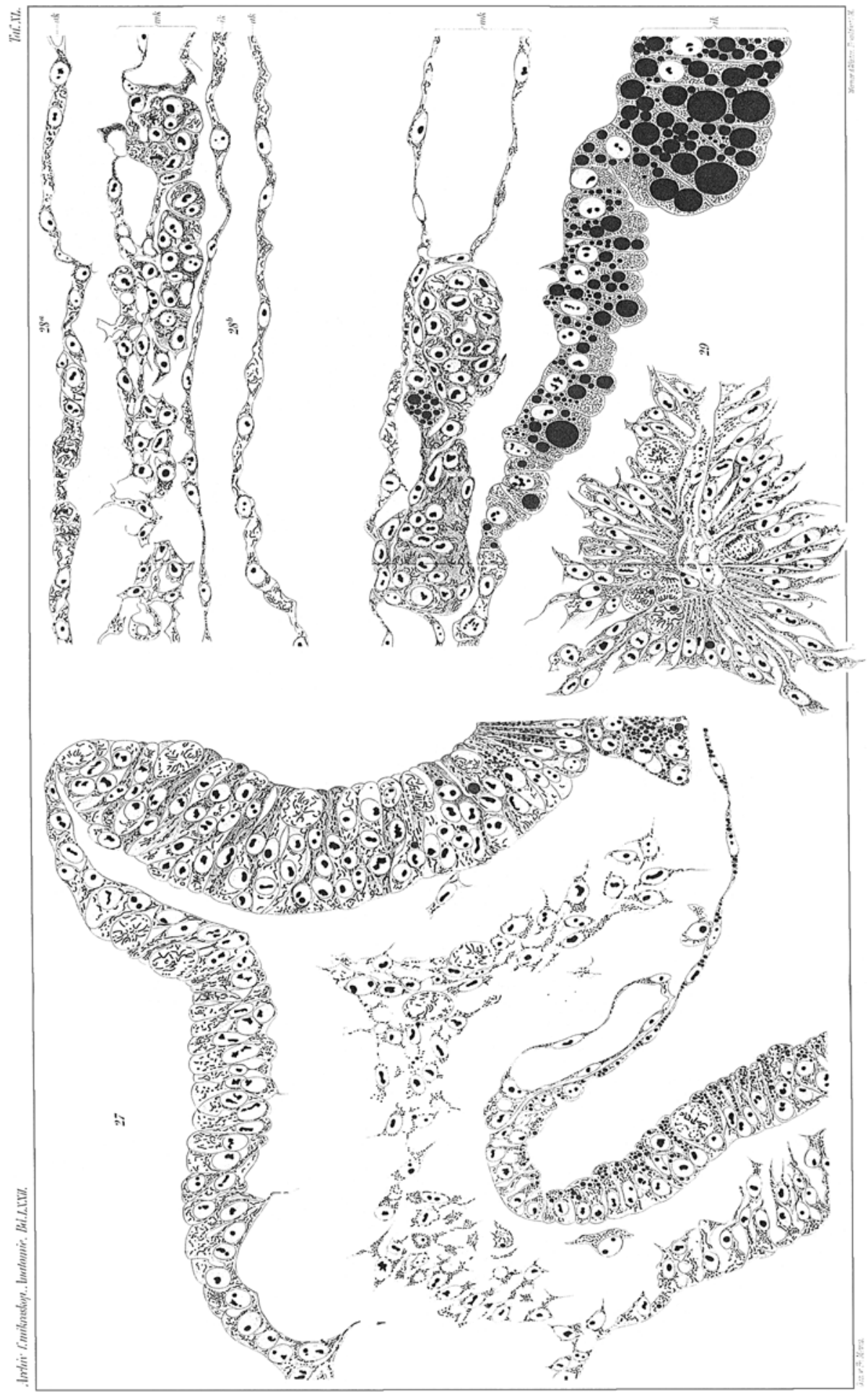




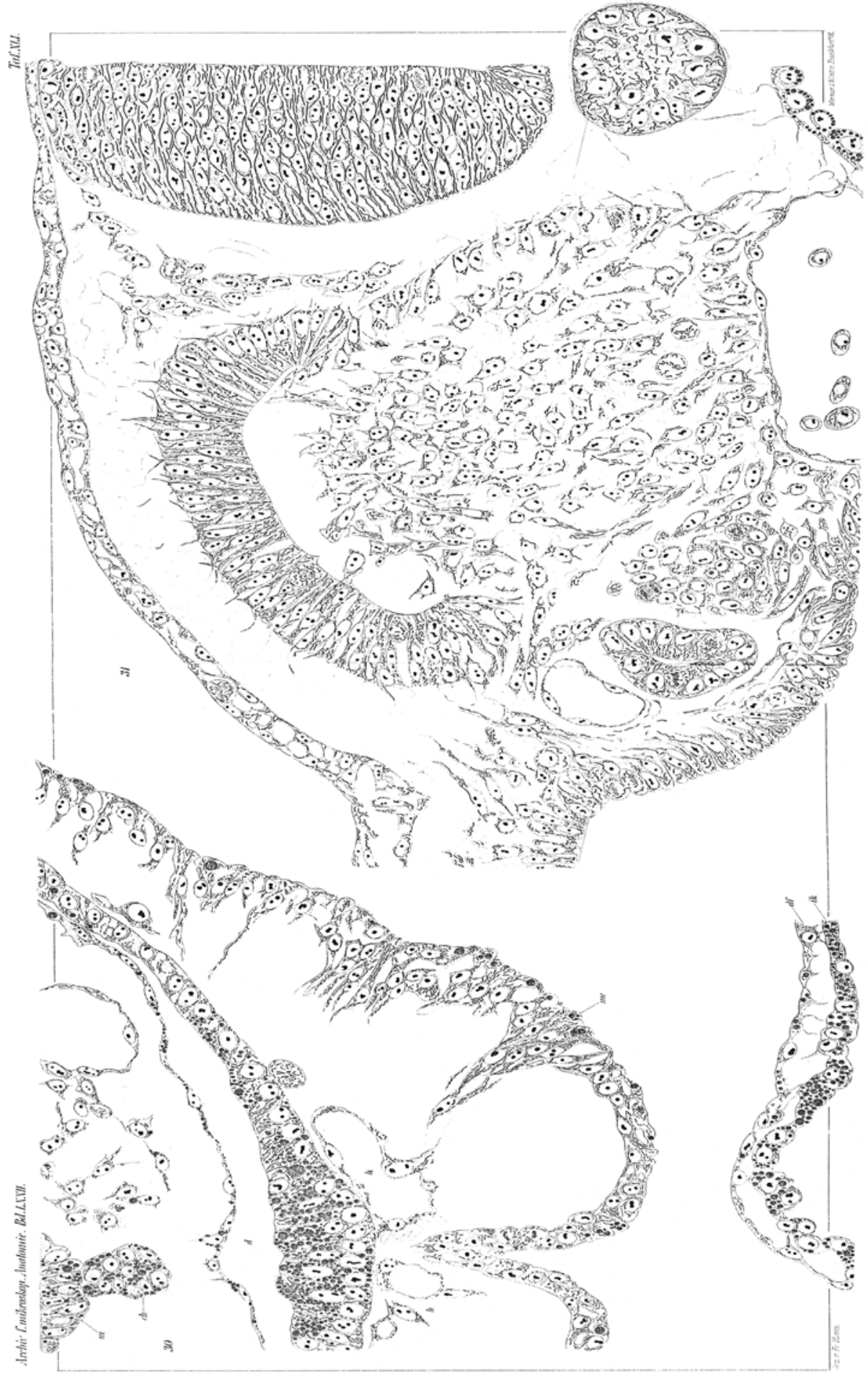




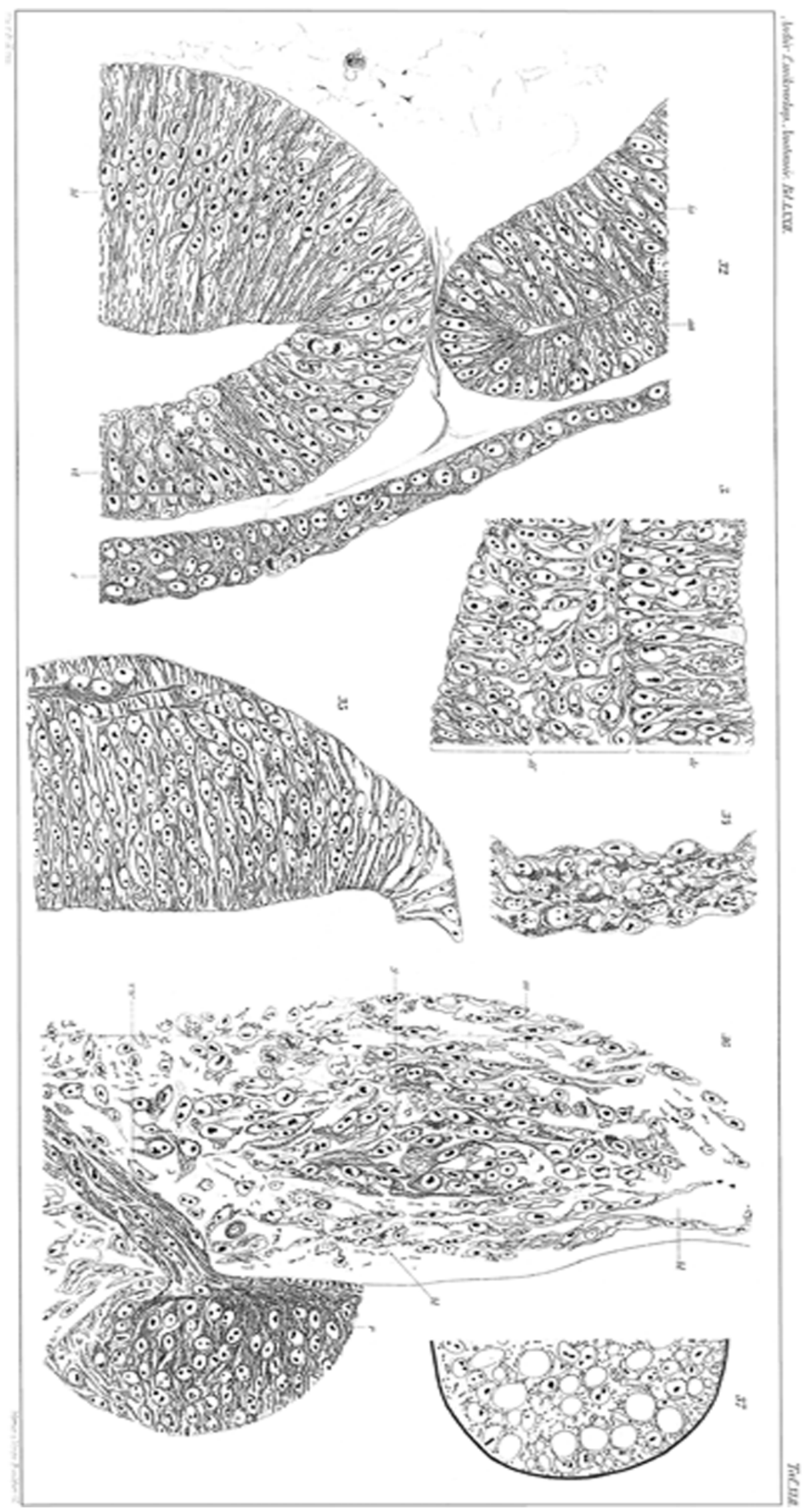

